\title{
, \\ Morphological Characteristics of Idiopathic Inflammatory Myopathies in Juvenile Patients
}

\author{
Anne Schänzer ${ }^{1, *+}{ }^{+}$, Leonie Rager ${ }^{1,+}{ }^{,}$, Iris Dahlhaus ${ }^{2}$, Carsten Dittmayer ${ }^{3}$, Corinna Preusse ${ }^{3,4}$, \\ Adela Della Marina ${ }^{5}{ }^{\circ}$, Hans-Hilmar Goebel ${ }^{3}$, Andreas Hahn ${ }^{6}$ and Werner Stenzel ${ }^{3}$
}

check for

updates

Citation: Schänzer, A.; Rager, L.; Dahlhaus, I.; Dittmayer, C.; Preusse, C.; Della Marina, A.; Goebel, H.-H.; Hahn, A.; Stenzel, W. Morphological Characteristics of Idiopathic Inflammatory Myopathies in Juvenile Patients. Cells 2022, 11, 109. https:// doi.org/10.3390/cells11010109

Academic Editor: Russell T. Hepple

Received: 18 November 2021

Accepted: 25 December 2021

Published: 30 December 2021

Publisher's Note: MDPI stays neutral with regard to jurisdictional claims in published maps and institutional affiliations.

Copyright: (c) 2021 by the authors Licensee MDPI, Basel, Switzerland. This article is an open access article distributed under the terms and conditions of the Creative Commons Attribution (CC BY) license (https:// creativecommons.org/licenses/by/ $4.0 /)$.
1 Institute of Neuropathology, Justus Liebig University, 35392 Giessen, Germany; leonie.j.rager@med.uni-giessen.de

2 Institute of Medical Informatics, Charité-Universitätsmedizin Berlin, Corporate Member of Freie Universität Berlin und Humboldt-Universität zu Berlin, 10117 Berlin, Germany; iris.dahlhaus@charite.de

3 Department of Neuropathology, Charité-Universitätsmedizin Berlin, Corporate Member of Freie Universität Berlin and Humboldt-Universität zu Berlin, 10117 Berlin, Germany; carsten.dittmayer@charite.de (C.D.); corinna.preusse@charite.de (C.P.); hans-hilmar.goebel@charite.de (H.-H.G.); werner.stenzel@charite.de (W.S.)

4 Department of Neurology with Institute of Translational Neurology, University Hospital Münster, 45147 Münster, Germany

5 Department of Pediatric Neurology, Centre for Neuromuscular Disorders, Centre for Translational Neuro-und Behavioral Sciences, University Duisburg-Essen, 45122 Essen, Germany; adela.dellamarina@uk-essen.de

6 Department of Child Neurology, Justus Liebig University, 35392 Giessen, Germany; andreas.hahn@paediat.med.uni-giessen.de

* Correspondence: anne.schaenzer@patho.med.uni-giessen.de; Tel.: +49-641-9941184; Fax: +49-99641-41189

+ These authors contributed equally to this work.

\begin{abstract}
Background: In juvenile idiopathic inflammatory myopathies (IIMs), morphological characteristic features of distinct subgroups are not well defined. New treatment strategies require a precise diagnosis of the subgroups in IIM, and, therefore, knowledge about the pathomorphology of juvenile IIMs is warranted. Methods: Muscle biopsies from 15 patients (median age 8 (range 3-17) years, $73 \%$ female) with IIM and seven controls were analyzed by standard methods, immunohistochemistry, and transmission electron microscopy (TEM). Detailed clinical and laboratory data were accessed retrospectively. Results: Proximal muscle weakness and skin symptoms were the main clinical symptoms. Dermatomyositis (DM) was diagnosed in 9/15, antisynthetase syndrome (ASyS) in $4 / 15$, and overlap myositis $(\mathrm{OM})$ in $2 / 15$. Analysis of skeletal muscle tissues showed inflammatory cells and diffuse upregulation of MHC class I in all subtypes. Morphological key findings were COX-deficient fibers as a striking pathology in DM and perimysial alkaline phosphatase positivity in anti-Jo-1-ASyS. Vascular staining of the type 1 IFN-surrogate marker, MxA, correlated with endothelial tubuloreticular inclusions in both groups. None of these specific morphological findings were present in anti-PL7-ASyS or OM patients. Conclusions: Morphological characteristics discriminate IIM subtypes in juvenile patients, emphasizing differences in aetiopathogenesis and supporting the notion of individual and targeted therapeutic strategies.
\end{abstract}

Keywords: myositis; dermatomyositis; muscle pathology; overlap myositis; juvenile; anti-synthetase syndrome

\section{Introduction}

Idiopathic inflammatory myopathy (IIM) is the most common form of myopathy in adult patients. Based on new insights in pathogenesis, it has become clear that individual immunomodulatory therapy strategies may be helpful for patients who do not respond sufficiently to current standard therapy [1,2]. Classification of IIM has been based on clinical, serological, and morphological features that lead to further definition of distinct subgroups, such as dermatomyositis (DM), antisynthetase syndrome (ASyS), immune-mediated necrotizing myopathy (IMNM), and sporadic inclusion body myositis (sIBM) [3-6]. Additionally, 
myositis can be associated with various rheumatologic diseases and is then named overlap myositis (OM) [7,8]. The currently used classification of myositis, based on pathophysiological and immunological insights, suggests that subtype-specific therapy may be the best treatment option [9].

Juvenile idiopathic inflammatory myopathy (jIIM) has many features in common with adult IIM. Both age groups harbor distinct clinical phenotypes, autoantibody findings, and associated outcomes [10-13]. However, a stringent classification of jIIM, including clinical, serological, and morphological features, similar to the one in adult patients, does not exist. This results in uncertainty when assigning children with myositis to different subgroups, and makes interpretation of study results and assessment of outcomes, including therapy failure, difficult. Myositis specific antibodies (MSAs) are implemented to diagnose juvenile IIM, allowing the delineation of distinct subtypes, such as DM, ASyS, and necrotizing myopathy (IMNM). Dependent on the respective studies, up to 50-90\% of the patients show that MSAs with the most frequent antibodies are anti-MDA5, anti-NXP2, and antiTIF-1 $\gamma[11,13-16]$.

Myositis, in conjunction with skin lesions, is also seen in other rheumatologic disorders, such as systemic lupus erythematosus (SLE), connective tissue diseases, and some monogenic autoinflammatory diseases, further impeding the diagnosis and treatment of jIIM [14,17-20]. Moreover, in jIIM patients with negative autoantibodies, finding a precise diagnosis can be problematic (although it is currently not known whether autoantibodies have the same relevance in children as in adults).

Juvenile DM is the most common form of jIIM affecting 2-4/100,000 children per year [11,21]. JDM can start as early as two years, and girls are more often affected than boys [10,22]. Clinical key features are skeletal muscle weakness with signs of inflammation on MRI and muscle biopsy, skin lesions, and systemic organ involvement. Phenotype and prognosis are highly variable [23]. Lipodystrophy and calcinosis uncommon in adult DM are frequently reported in jDM [24]. MSA can be detected in approximately $70 \%$ of patients. NXP-2 and TIF1 $\gamma$ antibodies are most frequent, followed by MDA5 and Mi-2 [25-27] antibodies. With the introduction of corticosteroids, mortality has dropped to 2-3\% [13]. However, steroid resistance in some patients results in poor prognosis and warrants new therapeutic strategies [28]. IFN-related endothelial injury and Janus kinase (JAK) inhibition has emerged as a novel therapeutic strategy in difficult-to-treat cases [1,29,30].

Clinical characteristics, MSA profiles, and muscle pathology are well described in adult DM, but can be different in jDM (for review see Tanboon et al., 2020 [6]) [3,31,32]. These differences are not fully explained and may reflect different modifying factors in jDM and aDM [22]. Muscle biopsies show the morphological hallmarks of perifascicular pathology and inflammatory cells with a high variability of pathology in both adult and juvenile DM [25,26,33]. However, distinct morphological features in MSA-defined DM subgroups and association with clinical phenotypes are mainly described in aDM [11,26].

Autoantibodies against the cytoplasmatic aminoacyl-tRNA synthetase (ARS) are associated with ASyS [34-36]. The frequency of ARS antibodies and, hence, the anti-synthetase syndrome are much lower in children (1-3\%) compared to adults (30-40\%) [13,37]. Clinical manifestation is similar in all age groups often associated with interstitial pneumonia [38]. The morphological hallmark of ASyS is a necrotizing perifascicular myositis with strong staining of the fragmented perimysium in alkaline phosphatase preparations in Jo1-associated ASyS [39,40]. Additionally, myonuclear actin filament inclusions are a unique morphological feature in adult ASyS [41].

Finally, IMNM is associated with severe muscle weakness, high creatine kinase levels, no or little skin involvement, and scattered necrotic and regenerating fibers at muscle biopsy $[42,43]$. IMNM is paradigmatically associated with anti-HMCGR or anti-SRP autoantibodies. IMNM is rare in children accounting for $1-4 \%$ of all jIIM cases [13,14,44,45].

In this single tertiary center study, we performed a detailed semi-quantitative analysis of muscle biopsies from fifteen children diagnosed with jIIM and seven non-diseased controls based on enzymatic stains, immunohistochemistry, and electron microscopy, and 
compared the morphological findings with clinical and laboratory data. The aim of the study was to identify morphological key findings which discriminate the different subtypes of juvenile IIM.

\section{Materials and Methods}

\subsection{Subjects and Samples}

Fifteen juvenile patients with the clinical diagnosis of myositis were included in the study. Patients were recruited from the department of pediatric neurology, Giessen, between 2002 and 2018. An open muscle biopsy was performed on all patients. Clinical and laboratory data were retrospectively analyzed in the database. Controls were obtained from seven age-matched children, who received a muscle biopsy because of suspected neuromuscular disorder or metabolic disease confirmed by biopsy and further clinical follow-up. All control muscle biopsies did not show any overt pathological changes, including thorough studies of immune phenomena. The median age at biopsy was 12 (9-17) years, 33\% female.

\subsection{Muscle Pathology Analysis}

\subsubsection{Histochemical, Enzymatic and Immunohistochemical Microscopy}

The unfixed muscle tissue was snap-frozen, and 6- $\mu$ m cryosections were processed for histochemical and enzymatic staining, according to standard procedures including hämalum eosin (H\&E) combined cytochrome-c-oxidase (COX)-succinate dehydrogenase (SDH), Gömöri trichrome, and alkaline phosphatase (ALP) [46]. Immunohistochemical stains were performed on cryosections using a Bench Mark XT automatic staining platform (Ventana, Heidelberg, Germany) with the following primary antibodies: mouse monoclonal anti-LC3 (0231-100/LC3-5F10, nanoTools, 1:100, Teningen, Germany); mouse monoclonal anti-p62 (610832, BD Biosciences, 1:500, Franklin Lakes, NJ, USA); mouse monoclonal anti-MHC class I (M0736, DAKO, 1:500); mouse monoclonal anti-MHC class II (M0775, DAKO, 1:200); mouse monoclonal anti-c5b-9 (MO777, DAKO, 1:50); mouse monoclonal anti-CD 31 (MO823, DAKO, 1:500); rabbit polyclonal anti-MxA2 (sc-50509, Santa Cruz, 1:100); mouse monoclonal anti-VEGF (sc 7269, Santa Cruz, 1:100); mouse monoclonal anti-Myotilin (RS034, Novocastra, 1:50); mouse monoclonal anti-MHCD (RNMy2/9D2, Novocastra, 1:20); mouse monoclonal anti-CD56 (18-0152, Invitrogen); mouse monoclonal anti-CD68 (M0814, DAKO, 1:100); mouse monoclonal anti-CD20 (M0755, DAKO, 1:100); mouse monoclonal anti-CD8 (M7103, DAKO, 1:500); and rabbit- monoclonal anti- CD3 (RM-9107-S0, Medac, 1:200) (Supplementary Table S1). Sections were examined with a Nikon eclipse 80i microscope, equipped with a Nikon digital camera DS-Fi1.

The muscle pathology was scored semi-quantitatively according to a modified consensus ranking scale (VAS) aimed at determining the severity of tissue damage published by Wedderburn and Varsani et al. $[47,48]$, including the following prominent domains: inflammation, vascular, muscle fibers, and connective tissue. Muscle pathology was rated from normal or absent (0) to strongly affected (10) on H\&E and Gömöri trichrome stained sections. Additionally, the muscle morphology was rated from normal or absent (0) to strong (3) for perifascicular atrophy, punched-out vacuoles (POV), necrotic muscle fibers, regenerative muscle fiber, oedema, and inflammatory cells using H\&E stained sections. ALP staining of fragmented perimysial cells and COX-negative or reduced (pale-blueish) fibers were estimated in standard stains. Antibody-stained sections were rated from physiological expression or absent (0) to strong (3). A reduction in vessel density was rated on anti-CD31 stained sections from normal (0) to strong (3). Three investigators (AS, LR, and WS) were blinded to the specimens during examination. Both AS and WS are experienced with semi-quantitative analysis of muscle sections and have performed similar studies previously [7,49-51]. 


\subsubsection{Transmission Electron Microscopy (TEM)}

Samples for TEM analysis were available for 13/15 patients. Small samples were taken from open muscle biopsies, fixed in $4 \%$ glutaraldehyde/0.4 MPBS, and processed according to standard procedures. For contrast in transmission electron microscopy (TEM), ultrathin sections were treated with $3 \%$ lead citrate- $3 \mathrm{H}_{2} 0$ with a Leica EM AC20 (ultrastain kit II) and examined at a Zeiss EM109 TEM, equipped with a sharp eye digital camera. Muscle pathology was scored from normal or absent (0) to strong (3), and myofibrillar disintegration, Z-band alterations, and glycogen deposits were analyzed in longitudinal sections at magnification 12,000 $\times$. Mitochondrial pathology and tubuloreticular deposits (TIR) in endothelial cells of endomysial capillaries were analyzed in cross sections at magnification $12,000 \times$. At least 10 endomysial capillaries in each specimen were analyzed. The presence of deposits was rated from 0 to 3. Myonuclear actin inclusions were studied in selected cases with ASyS (P4, P14, P6). At minimum, 200 nuclei were analyzed in each specimen.

\subsection{Statistical Analysis}

To determine whether there were differences among jIIM subgroups DM, ASyS, and $\mathrm{OM}$, with respect to their morphological characteristics (semi-quantitative variables), the Kruskal-Wallis test was performed. Age at biopsy, VAS score, and laboratory parameters were also compared using the Kruskal-Wallis test. All $p$-values were adjusted for multiple testing. $\mathrm{R}$ version 4.0 .3 was used to perform the analysis.

\section{Results}

\subsection{Clinical Data}

Fifteen patients were included in the study. Median age at biopsy was 8 (range 3-17) years, of whom of $73 \%$ were females (Figure 1A). Duration of disease was subacute ( $>14$ days) in the majority of patients. Dermatological manifestation (100\%), muscle weakness (93\%), and myalgia $(73 \%)$ were the key diagnostic elements. Muscle weakness was particularly present in the proximal lower limbs with difficulties in climbing stairs or getting up from the chair. Fatigue $(47 \%)$ and mood swing with sadness $(33 \%)$ were reported. Four patients $(27 \%)$ developed calcinosis. Extramuscular manifestation with pulmonary $(20 \%)$ and cardiac involvement $(27 \%)$ occurred (Tables 1 and S2). Specific EMG signs, characteristic of dermato-polymyositis, such as abundant abnormal spontaneous activity (e.g., small positive waves firing at slow rates, defibrillation potentials, myotonic discharges), in conjunction with short polyphasic muscle unit potentials of low amplitude, were detected in $72 \%$. Rather unspecific alterations, such as rare spontaneous activity and a mixture of some polyphasic low-amplitude and normal muscle unit potentials, were present in $27 \%$. Typical muscle MRI findings of dermato-polymyositis, such as marked T2 hyperintensity in the thigh muscles with edema on T2-fat suppressed sequences and marked contrast enhancement on post-gadolinium sequences, were detected in $73 \%$. Characteristic ultrasound abnormalities, such as diffusely increased echogenicity and blurred fibrillar echotexture of the muscle, as well as thickened fascia, were seen in $67 \%$ (Tables 1 and S4). Figure 2 shows characteristic skin involvement (A, B) and MRI findings (C-E) of patients with IIM.

Out of 12 children, $6(50 \%)$ had autoantibodies, with anti-NXP-2 (2/12), anti-Jo1 (2/12), and anti-PL-7 (2/12). In two patients, anti-nRNP/Sm antibodies were detected. Anti-nuclear antibodies (ANA) were positive in $69 \%$. Elevated serum CK and LDH levels were the most prominent laboratory features (Figure 1C,D). ASyS patients generally showed higher CK levels compared to the DM and OM groups but did not reach statistical significance $(p>0.05)$ (Figure 1C,D). Extramuscular symptoms did not occur in anti-PL7-ASyS and OM. Comorbidities were higher in anti-PL-7-ASyS and OM. Accompanying symptoms, such as fatigue, was present in all subgroups. Myalgia was not noted in the two patients with anti-Jo1-ASyS (Supplemementary Table S3). Muscle biopsies had been taken before start of therapy in 13/15 patients. Only P13 and P4 received pulse steroid therapy shortly before biopsy. 


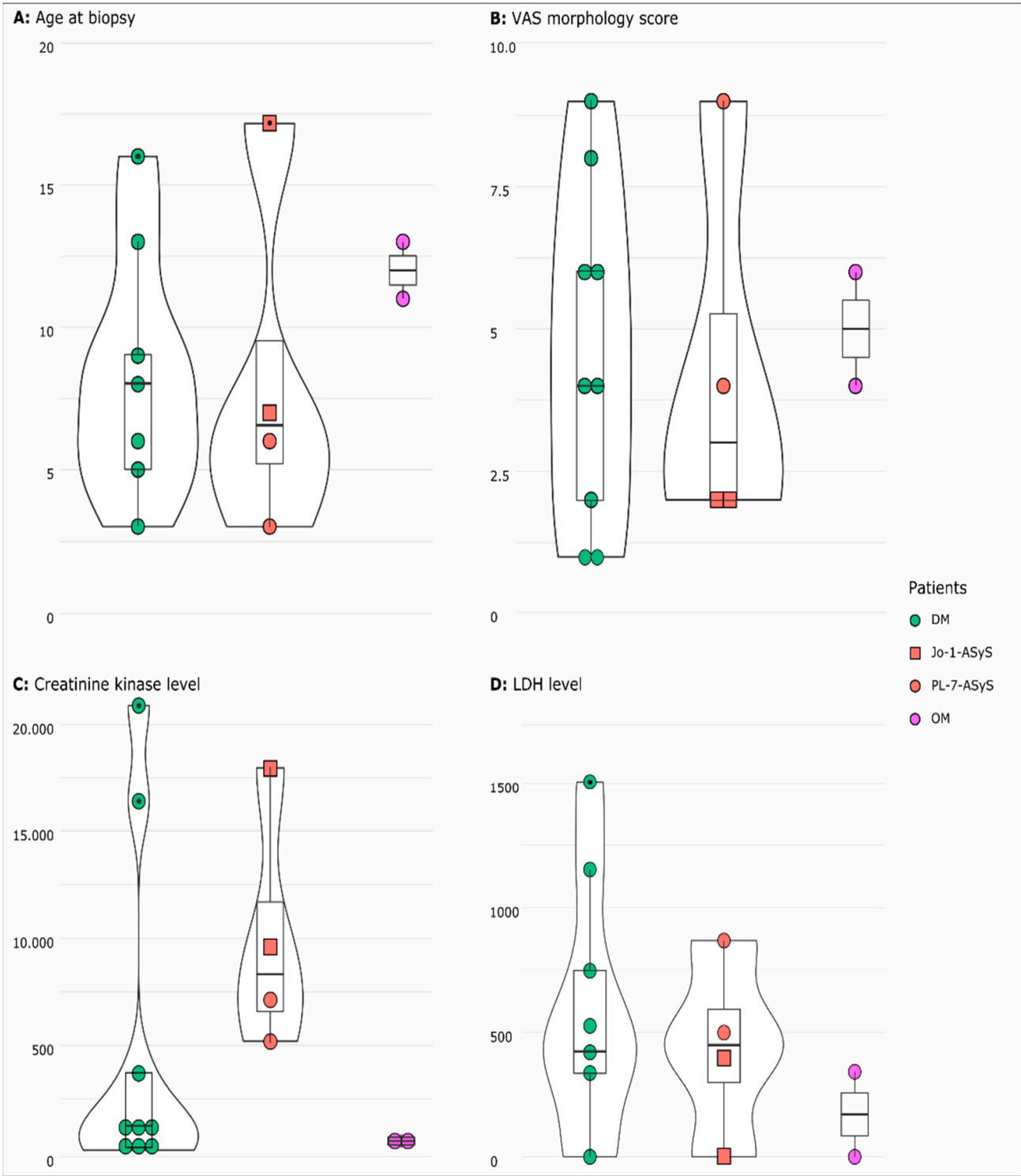

Figure 1. Distribution as violin plot (rotated kermel density curve and boxplot) of age at biopsy (A) VAS morphology score (B), serum creatine kinase (C), and LDH (D) levels in jIIM subgroups DM, ASyS (Jo-1, PL-7), and OM. Wide sections represent a higher probability that the patients take the value and small sections represent a lower probability. The box represents the interquartile range, the whiskers minimum $(\mathrm{Q} 1-1.5 \times \mathrm{IQR})$ and maximum $(\mathrm{Q} 3+1.5 \times \mathrm{IQR})$, and the points show the outliers. For all parameters, the $p$-value did not reach statistical significance. (DM = dermatomyositis; ASyS = antisynthetase syndrome; $\mathrm{OM}=$ overlap myositis). 


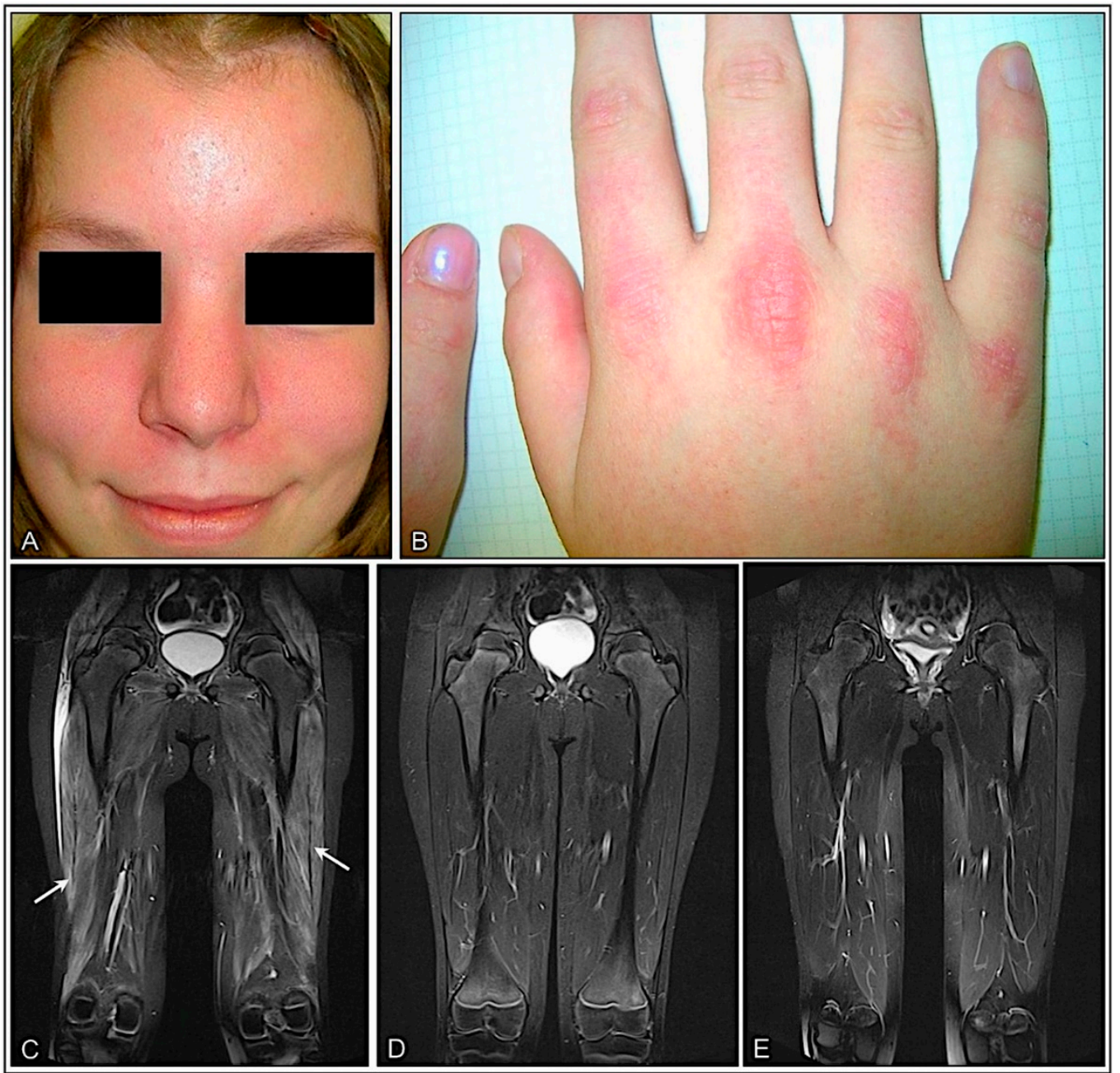

Figure 2. Proximal myopathy and skin lesions are the clinical hallmarks in patients with jIIM. Mild facial erythema (A) and discrete erythematous papules overlying the metacarpal joints (Gottron's papules) (B) in a 16-year-old girl with DM (P5). Coronal MR images in a 10-year-old patient with anti-NXP2 antibody-associated DM (P4) showing mild contrast enhancement in T1-weighted images and diffuse oedema of the thigh muscles at first presentation (arrows) (C) resolving completely with treatment after 6 (D) and 12 months (E).

Table 1. Summary of demographic data of patients with jIIM.

\begin{tabular}{cc}
\hline Patients with jIIM & \\
\hline Number of patients & 15 \\
Age at biopsy (median) & range \\
female & 8 years \\
Skeletal muscle symptoms & 17 years) \\
Myalgia & $\mathbf{1 4} \%)$ \\
\hline Exercise induced myalgia & $11(73 \%)$ \\
Proximal weakness & $6(40 \%)$ \\
\hline Skin symptoms & $14(93 \%)$ \\
\hline Dry skin & $\mathbf{1 5 / 1 5 ( 1 0 0 \% )}$ \\
Exanthema & $6(40 \%)$ \\
Erythema & $3(20 \%)$ \\
Butterfly rash & $4(27 \%)$ \\
\hline
\end{tabular}


Table 1. Cont.

\begin{tabular}{|c|c|}
\hline Skin symptoms & $15 / 15(100 \%)$ \\
\hline Redness, livid coloration & $8(53 \%)$ \\
\hline Raynaud's phenomenon & $1(7 \%)$ \\
\hline Gottron's papules & $8(53 \%)$ \\
\hline Nail fold changes & $1(7 \%)$ \\
\hline Calcinosis & $4(27 \%)$ \\
\hline Accompanying symptoms & $15 / 15(100 \%)$ \\
\hline Fever & $3(20 \%)$ \\
\hline Difficulty swallowing & $1(7 \%)$ \\
\hline Morning stiffness & $1(7 \%)$ \\
\hline Arthritis & $1(7 \%)$ \\
\hline Weight gain & $2(13 \%)$ \\
\hline Weight loss & $1(7 \%)$ \\
\hline Night sweat & $1(7 \%)$ \\
\hline Oedema & $3(20 \%)$ \\
\hline Lymphadenopathy & $1(7 \%)$ \\
\hline Fatigue, concentration difficulties & $7(47 \%)$ \\
\hline Loss of appetite/rejection to drink & $1(7 \%)$ \\
\hline Sadness/mood slump/mood swings & $5(33 \%)$ \\
\hline Social withdrawal & $3(20 \%)$ \\
\hline Extramuscular symptoms & $5 / 15(33 \%)$ \\
\hline Pulmonary restriction & $2(13 \%)$ \\
\hline Pneumonia & $1(7 \%)$ \\
\hline Cardial involvement & $4(27 \%)$ \\
\hline Comorbidities & $5 / 15(33 \%)$ \\
\hline Cystic fibrosis & $1(7 \%)$ \\
\hline Factor-V-Leiden mutation & $1(7 \%)$ \\
\hline Hypothyroidism & $1(7 \%)$ \\
\hline Steatosis hepatis & $1(7 \%)$ \\
\hline HLA-B27 enthesitis & $1(7 \%)$ \\
\hline Laboratory results & $15 / 15(100 \%)$ \\
\hline Creatine kinase $(\mathrm{CK}) \uparrow$ & $14(93 \%)$ \\
\hline Lactatdehydrogenase (LDH) $\uparrow$ & $11(73 \%)$ \\
\hline GOT $\uparrow$ & $11(73 \%)$ \\
\hline $\mathrm{GPT} \uparrow$ & $7(47 \%)$ \\
\hline \multicolumn{2}{|l|}{ Serum antibodies } \\
\hline Myositis-specific-antibodies (MSA) & $6 / 12(50 \%)$ \\
\hline Anti-NXP-2 & $2(17 \%)$ \\
\hline Anti-PL-7 & $2(17 \%)$ \\
\hline Anti-Jo-1 & $2(17 \%)$ \\
\hline Myositis-associated-antibodies (MAA) & $2 / 12(17 \%)$ \\
\hline Anti-Ro52 & $1(8 \%)$ \\
\hline PM75 & $1(8 \%)$ \\
\hline Antinuclear antibodies (ANA) & $9 / 13(69 \%)$ \\
\hline \multicolumn{2}{|l|}{ Systemic Lupus erythematodes (SLE) } \\
\hline Anti-double-stranded-DNA-antibodies (Anti-dsDNA) & $1(8 \%)$ \\
\hline Smith-Antibodies (Anti-Sm/Sm-AK) & $1(8 \%)$ \\
\hline Anti RNP/Sm-AK & $2(17 \%)$ \\
\hline
\end{tabular}


Table 1. Cont.

\begin{tabular}{cc}
\hline MRI skeletal muscle & $\mathbf{1 1 / 1 5}(\mathbf{7 3} \%)$ \\
\hline Normal & $2(18 \%)$ \\
Oedema & $6(54 \%)$ \\
Enhancement of contrast medium/signal alterations & $4(36 \%)$ \\
Compatible with myositis & $8(73 \%)$ \\
\hline Musculoskeletal Ultrasound & $\mathbf{9 / 1 5 ( 6 0 \% )}$ \\
\hline Oedema & $3(33 \%)$ \\
Enhancement of echogenicity/signal alterations & $6(67 \%)$ \\
Compatible with myositis & $6(67 \%)$ \\
\hline Electromyography (EMG) & $\mathbf{1 1 / 1 5}(\mathbf{7 3} \%)$ \\
\hline Myopathic, compatible with myositis & $8(72 \%)$ \\
Unspecific sign & $3(27 \%)$ \\
\hline
\end{tabular}

\subsection{Muscle Pathology}

3.2.1. Muscle Pathology Score and Inflammatory Cell Invasion Are Highly Variable in All IIM Subtypes

Seven non-diseased control muscle biopsies showed no significant pathology and served as a morphological baseline for standard enzymatic and immunohistochemical studies and VAS score (Supplementary Table S6).

The muscle pathology (severity) score VAS showed a high heterogeneity in all subgroups with the highest score of 9 present in an anti-NXP-2-DM patient and in an antiJo-1-ASyS patient. There was no significant difference regarding the overall pathological severity score between DM, ASyS, and OM ( $p=0.8979)$ (Figure 1B). Perifascicular atrophy and punched-out vacuoles (POV) were mainly seen in DM and anti-Jo-1-ASyS, whereas necrotic fibers, oedema, and regeneration were present in all IIMs. In two cases with OM, the VAS score was high (4-6) with many necrotic and regenerating fibers. P13, with the diagnosis of anti-NXP-2-DM, showed a weak pathology morphology in all sections, consistent with a mild clinical phenotype and normal CK levels (Figure 3, Table A1). Inflammatory cells were present in all IIM muscle specimens, presenting as mainly T-cells (CD3, CD8) and macrophages (CD68). B-cells (CD20) were present only in few cases of all subgroups (Figure 4, Table A2).

\subsubsection{COX Deficient Fibers Are a Striking Pathology in DM Biopsies}

Only in DM skeletal muscle biopsies, perifascicular COX-deficient fibers were detectable, albeit with a high variability from 0-3. COX-deficient fibers were absent in ASyS or OM cases (Table A1, Figure 3).

\subsubsection{Perimysial Alkaline Phosphatase (ALP) Positivity Is Specific for Anti-Jo-1-ASyS}

Alkaline phosphatase (ALP) positivity of fragmented perimysial tissue is a specific finding to discriminate ASyS cases from other IIM subtypes [41,52]. Strong perimysial staining with ALP was only present in two cases of anti-Jo-1-ASyS, but not in PL-7-ASyS or other IIM cases consistent with findings in adult patients [39] (Table A1, Figure 3).

\subsubsection{Sarcolemmal Upregulation of MHC Class I, MHC Class II and Sarcolemmal Complement Deposits}

MHC class I was strongly upregulated on the muscle fiber sarcolemma in almost every biopsy (14/15) of jIIM, confirming the diagnosis of myositis [53]. MHC1 showed a diffuse upregulation pattern in most cases with a perifascicular gradient of varying degree in the majority of DM and anti-Jo-1-ASyS. No perifascicular MHC class I upregulation was seen in $\mathrm{OM}$ and PL7-ASyS. MHC class II sarcolemmal upregulation was present in the majority of the cases but less strong with a scattered distribution in most cases, in line with previously shown results [53]. In two DM cases (P2, P12) with strong pathology, a perifascicular 
staining pattern of MHC class II was more pronounced. Sarcolemmal deposits (C5b-9) on muscle fibers were present in 3/9 DM, 2/4 ASyS, and 1/2 OM with the strongest expression in an anti-Jo-1-ASyS, predominately in the perifascicular region (Table A2, Figures 4 and 5).

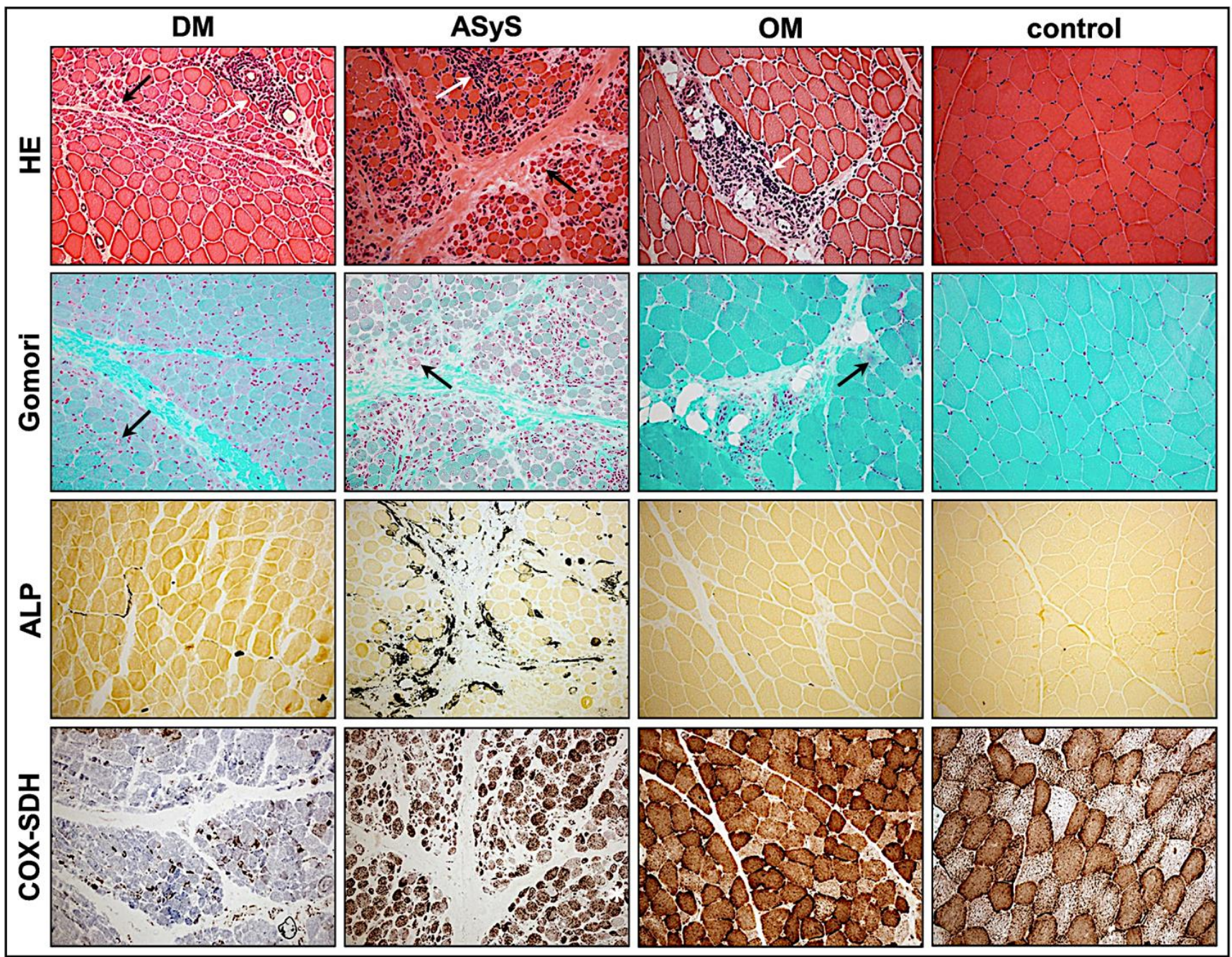

Figure 3. Representative staining of standard stained sections of jIIM from patients with DM (P4), anti-Jo1-ASyS (P14), OM (P3), and control. In H\&E-stained sections, perifascicular atrophy (black arrows) is prominent in biopsies from patients with DM and anti-Jo1-ASyS. In OM, atrophic fibers are distributed throughout the section. Inflammatory cell infiltrates (white arrows) are mainly located perifascicularly in DM, perifascicularly in anti-Jo1-ASyS, and perimysial in OM sections. In Gömori trichrome, muscle fibers show a strong alteration of myofibrillar structures in DM and anti-Jo1-ASyS (arrows) but not in OM. ALP is highly upregulated (black) in the perimysium of anti-Jo1-ASyS and shows some subtle upregulation in DM but not in OM. COX-negative fibers appear blue in the COX-SDH staining with an exclusively high number of COX-deficient fibers in the DM sections (magnification $20 \times$ ). (DM = dermatomyositis; ASyS = antisynthetase syndrome; $\mathrm{OM}=$ overlap myositis). 


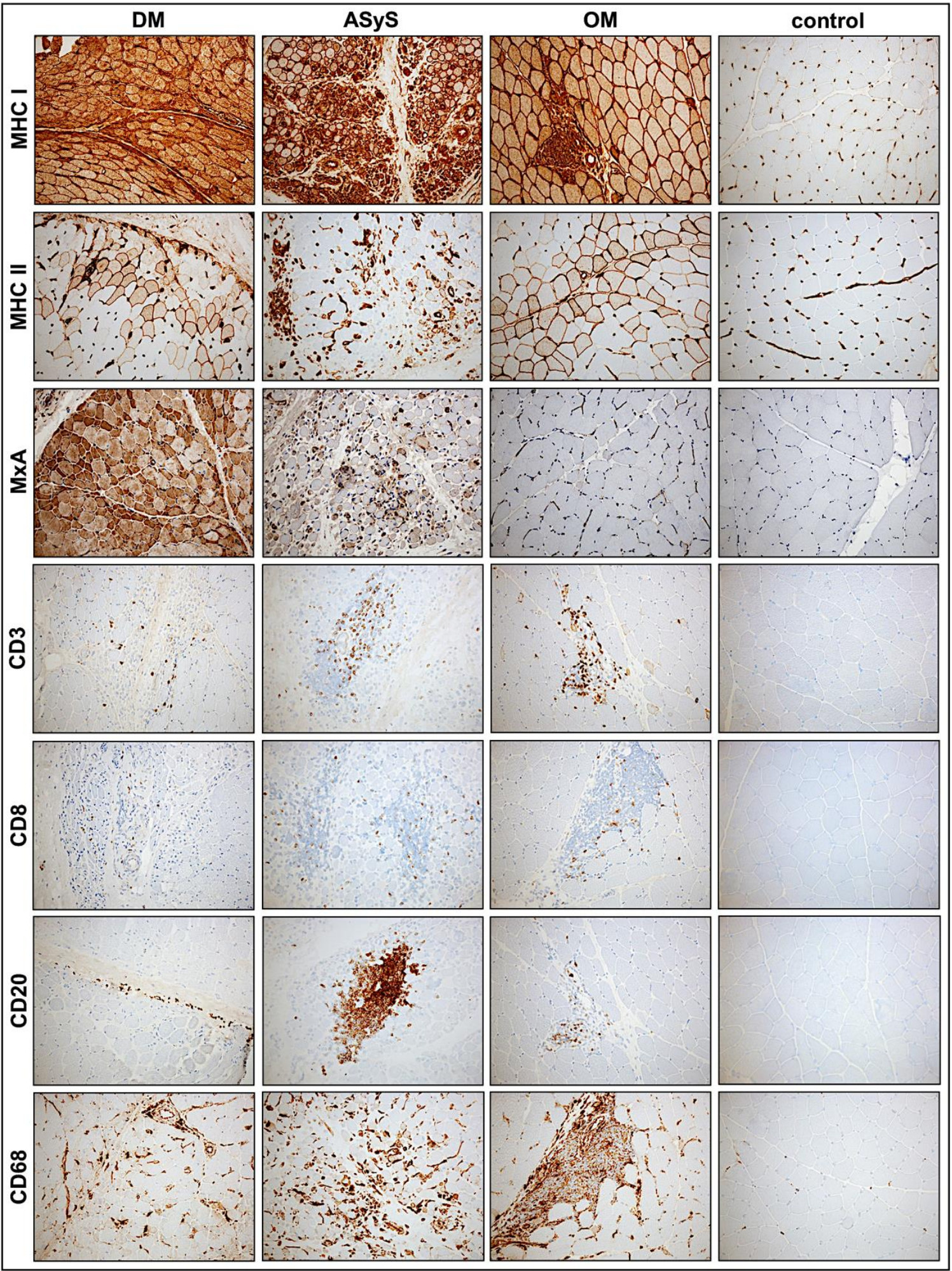

Figure 4. Representative staining of immunohistochemical features of biopsies from patients with DM (P4), anti-Jo1-ASyS (P14), OM (P3), and controls. The upregulation of MHC class 1 on muscle fibers is ubiquitously strong in all IIM subtypes, whereas MHC class II is mainly expressed at perifascicular muscle fibers. Upregulation of MxA is the highest in DM but also present in anti-Jo1ASyS. Inflammatory cells occur in all subtypes, mainly T-lymphocytes (CD3, CD8). B-lymphocytes (CD20) are prominent in Jo-1-ASyS. Macrophages (CD68) are prominent in all subtypes (magnification $20 \times)$. (DM = dermatomyositis; ASyS = antisynthetase syndrome; $\mathrm{OM}=$ overlap myositis $)$. 

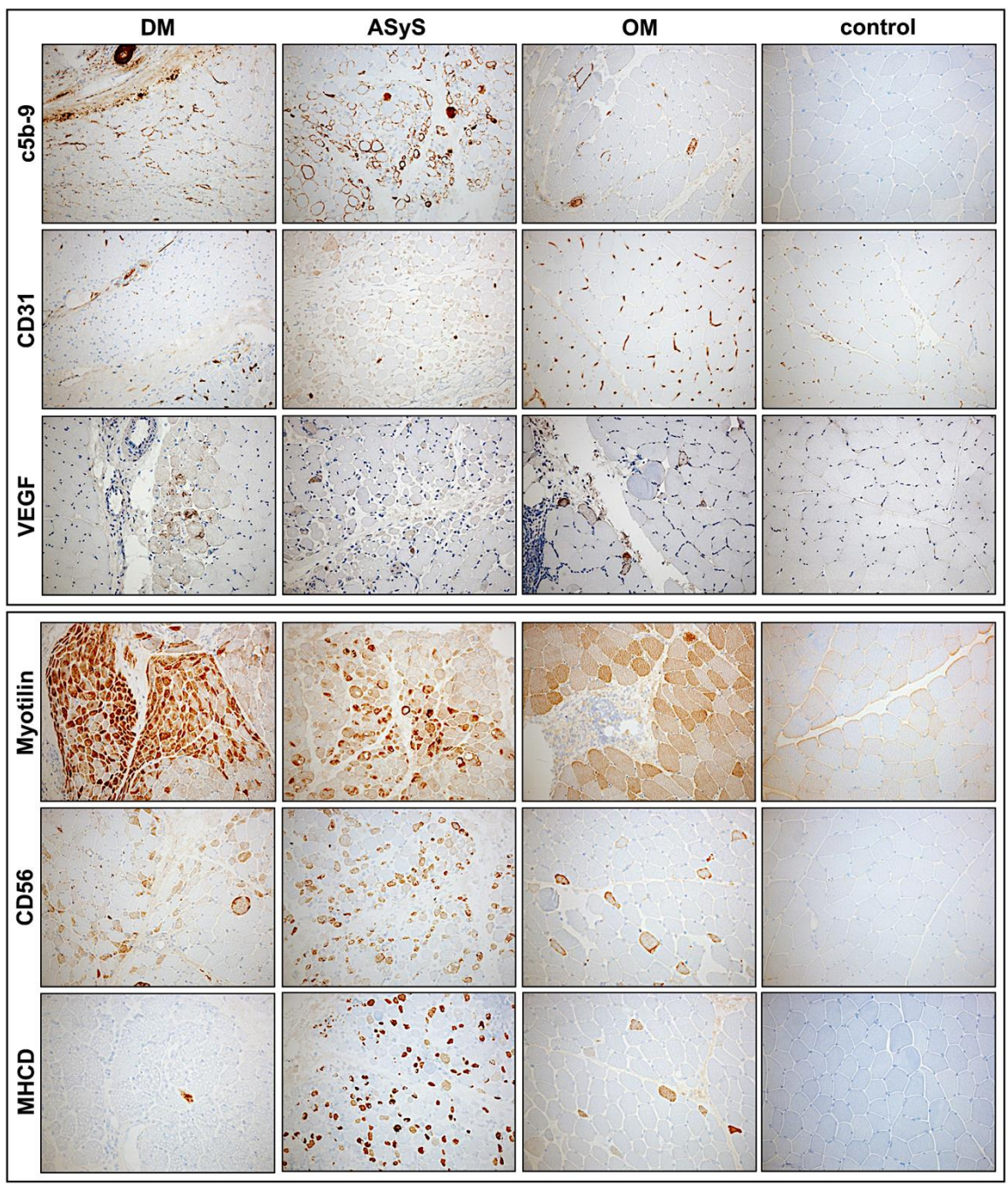

Figure 5. Representative stains of immunohistochemical features of biopsies from patients with DM (P4), anti-Jo1-ASyS (P14), OM (P3) and control. In DM, complement deposits (C5b-9) on endomysial capillaries are strong at the perifascicular region but can also be present in anti-Jo1-ASyS. Strong complement deposits on myofibers are present in anti-Jo1-ASyS in a perifascicular pattern. Few complement deposits are present on scattered fibers in $\mathrm{OM}$. CD $31^{+}$endomysial capillaries are reduced in DM and anti-Jo1-ASyS. Focal reduction in CD31+ vessels is also present in OM. Upregulation of vascular endothelial growth factor (VEGF) is mainly seen in DM but can also occur in anti-Jo1-ASyS and OM. Myotilin staining indicates a disorganization of sarcomeric structure. Strong perifascicular sarcomeric disruption is present in DM and less strong in anti-Jo1-ASyS. With antibodies against CD56 and MHCD, a strong regenerative capacity is seen in anti-Jo1-ASyS (magnification 20×). (DM = dermatomyositis; ASyS = antisynthetase syndrome; $\mathrm{OM}=$ overlap myositis).

\subsubsection{Vascular Pathology and Upregulation of Proangiogenic Factor VEGF}

Vascular pathology might contribute to muscle damage in jIIM. Strong complement deposits (C5b-9) were present on endomysial capillaries in DM (7/9) and anti-Jo-1-ASyS $(2 / 2)$, whereas no capillary deposits were detected in anti-PL-7-ASyS and OM (Table A2, Figure 5). A reduction in CD31+ endomysial capillaries was more prominent in DM and anti-Jo1-ASyS compared to anti-PL7-ASyS and OM. The proangiogenic factor VEGF was upregulated with perifascicular distribution in DM and anti-Jo-1-ASyS cases. Moderate rarefication of CD31+ capillaries, and VEGF upregulation were also present in OM (Table A2, Figure 5). 


\subsubsection{Ultrastructural Pathology}

Analyzing the muscle fiber at ultrastructural level shows disruption of myofibrillar architecture with a high variability in all subtypes (Table A3, Figure 6A-F). Mitochondria did show only mild pathology in few samples of IIM with subsarcolemmal aggregation, increased size in diameter (polymorphism), and disruption of cristae structure without any distinct differences between the subgroups (Table A3, Figure 6G-J). Analyses of three cases of ASyS revealed characteristic filamentous nuclear inclusions in 3/410 examined myonuclei, only in anti-Jo-1 ASyS (P14). Due to a section thickness of approximately $200 \mathrm{~nm}$, the substructure of inclusion appeared slightly more homogenous, as compared to 60-100-nm sections. No nuclear inclusion was found in ASyS-PL-7 (P6, P9) (Table A3, Figure $6 \mathrm{~K}-\mathrm{M})$.

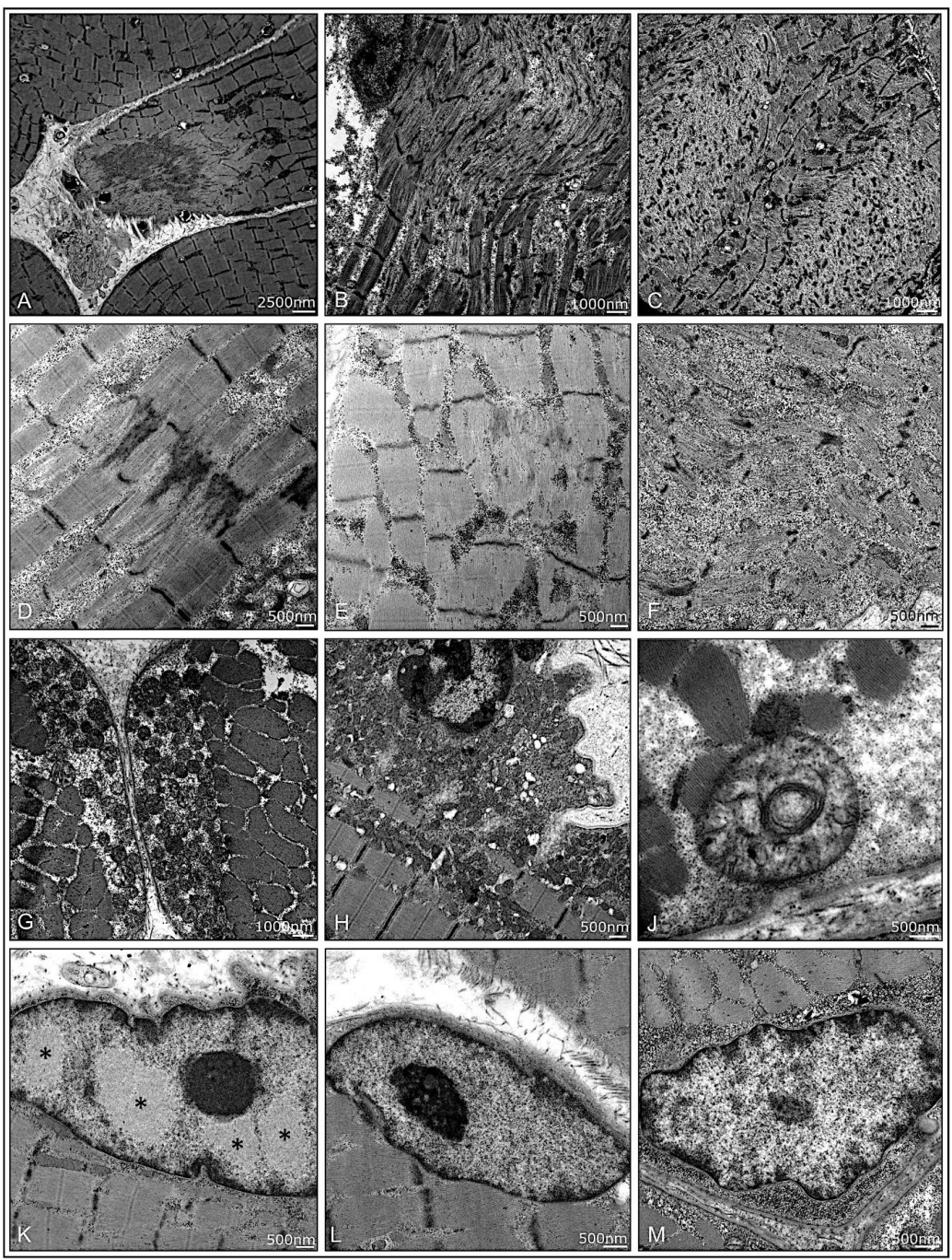

Figure 6. Ultrastructural pathology in muscle biopsies from patients with jIIM. A high variability of disruption of myofibrillar architecture can be present in all subtypes. Focal, core-like alterations of the sarcomeric architecture in DM (P10) (A), P4 (B), and P12 (C). Z-band alterations with Z-band streaming in P15 (D) and dissolving Z-bands in anti-Jo1-ASyS (P14) (E) and focal glycogen deposits in OM (P7) (F). Moderate mitochondrial pathology with some mitochondrial subsarcolemmal aggregation in PL-7-ASyS (P9) (G) and DM (P2) (H). Few mitochondria with increased variability of diameter and altered cristae structure in P9 (J). Myonuclei with characteristic filamentous nuclear inclusions were only found in anti-Jo1-ASys (P14); note the different patterns of euchromatin, heterochromatin, and the nucleolus, as compared to the nuclear inclusions $\left(^{*}\right)(\mathbf{K})$. No inclusions were found in PL-7-ASyS (P6, P9) (L,M). (DM = dermatomyositis; ASyS = antisynthetase syndrome; OM = overlap myositis). 
3.2.7. IFN 1 Surrogate Marker MxA Upregulation Correlates with Endothelial Inclusions

Myxovirus resistance protein A (MxA) is a type I-IFN-induced protein and serves as a diagnostic tool to discriminate DM from other IIM subtypes in juvenile and adults, which are at least equivalent in diagnostic performance compared to MHC class I staining [54]. In our study, MxA staining showed strong upregulation on the sarcolemma of myofibers and vessels with a perifascicular predominance distribution in $8 / 9$ patients with DM. The MxA expression correlated with the presence of endothelial tubuloreticular inclusions (TRIs) at TEM. In two patients with Jo-1-ASyS sarcoplasmic and vessel staining by MxA, an antibody was prominent, similar to those of the DM cases. TRIs were also frequent in one of the Jo-1-ASyS, and the only case of Jo-1-AsyS was available for TEM. No significant perifascicular MxA upregulation or endothelial TRI were found in patients with anti-PL-7ASyS $(n=2), O M(n=2)$, or in the controls $(n=7)$. Only few scattered fibers with weak MxA upregulation were detected in P6 (anti-PL-7-ASyS) and P3 (OM) (Table A3, Figure 7).

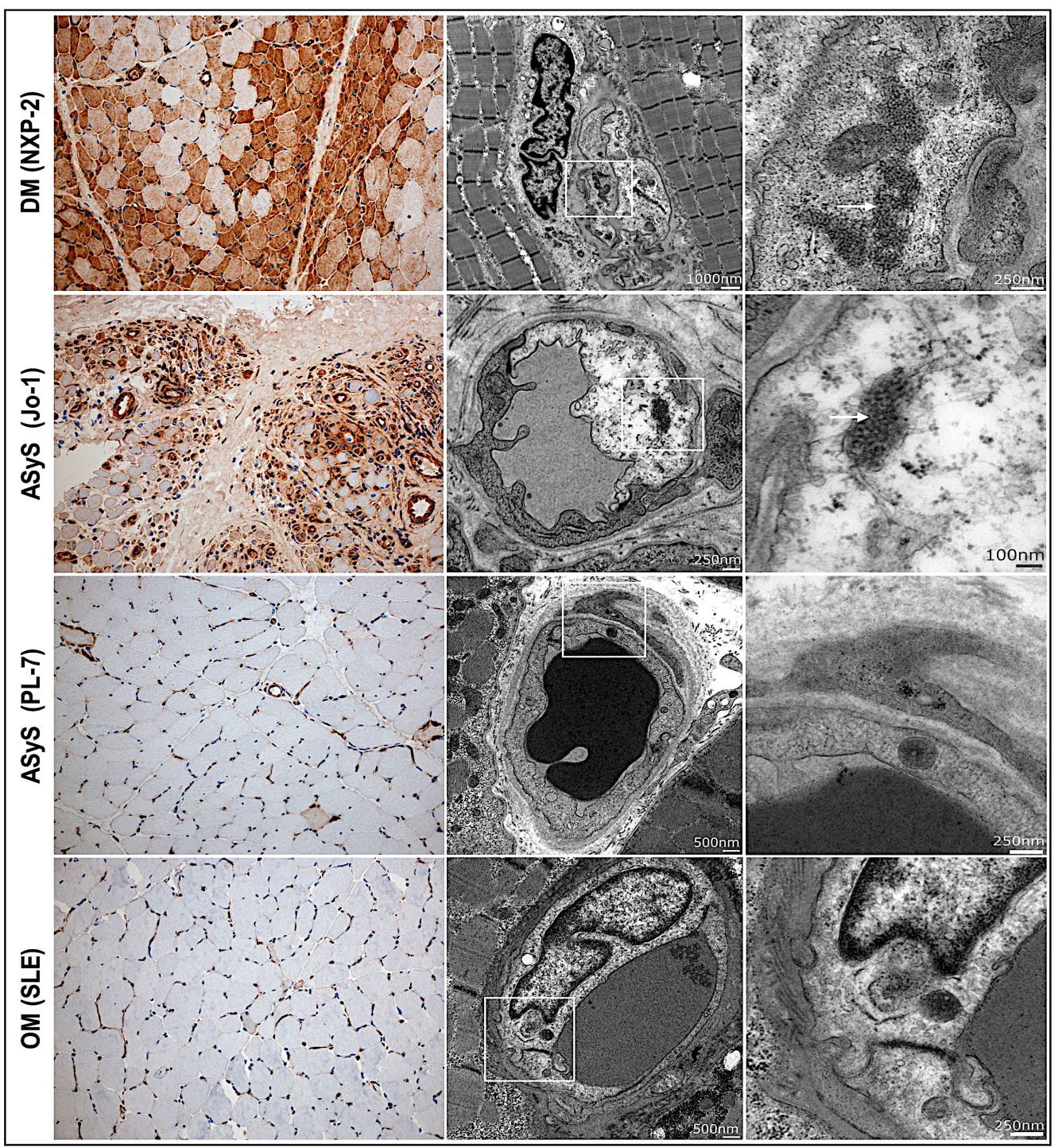

Figure 7. MxA upregulation and tubuloreticular inclusions in muscle biopsies from patients with IIM. Strong perifascicular upregulation of interferon surrogate marker MxA in DM (P4) and anti-Jo-1 ASyS (P14) associated with tubuloreticular inclusions (TIR) in the endothelial cells of small vessels. Weak MxA upregulation on scattered myofibers with nonspecific osmiophilic inclusions in anti-PL7 ASyS (P6) and OM (P3) (magnification 20×) (DM = dermatomyositis; ASyS = antisynthetase syndrome; $\mathrm{OM}=$ overlap myositis). 


\subsubsection{Moderate Activation of Autophagy in jIIM}

We analyzed the expression of the prototypic autophagy-related markers, $\mathrm{p} 62$ and LC3, in muscle biopsies from jIIM patients compared to controls. Few LC3 or p62-positive inclusions (rating from $0.5-1$ ) were shown in $5 / 15$ biopsies. In sections with a higher score (P12-score 8, P4-score 9, P14-score 9), LC3 expression was more pronounced. Additionally, in these cases, the muscle fiber regeneration (CD56, MHCD) was higher (Table A3, Figure 8).

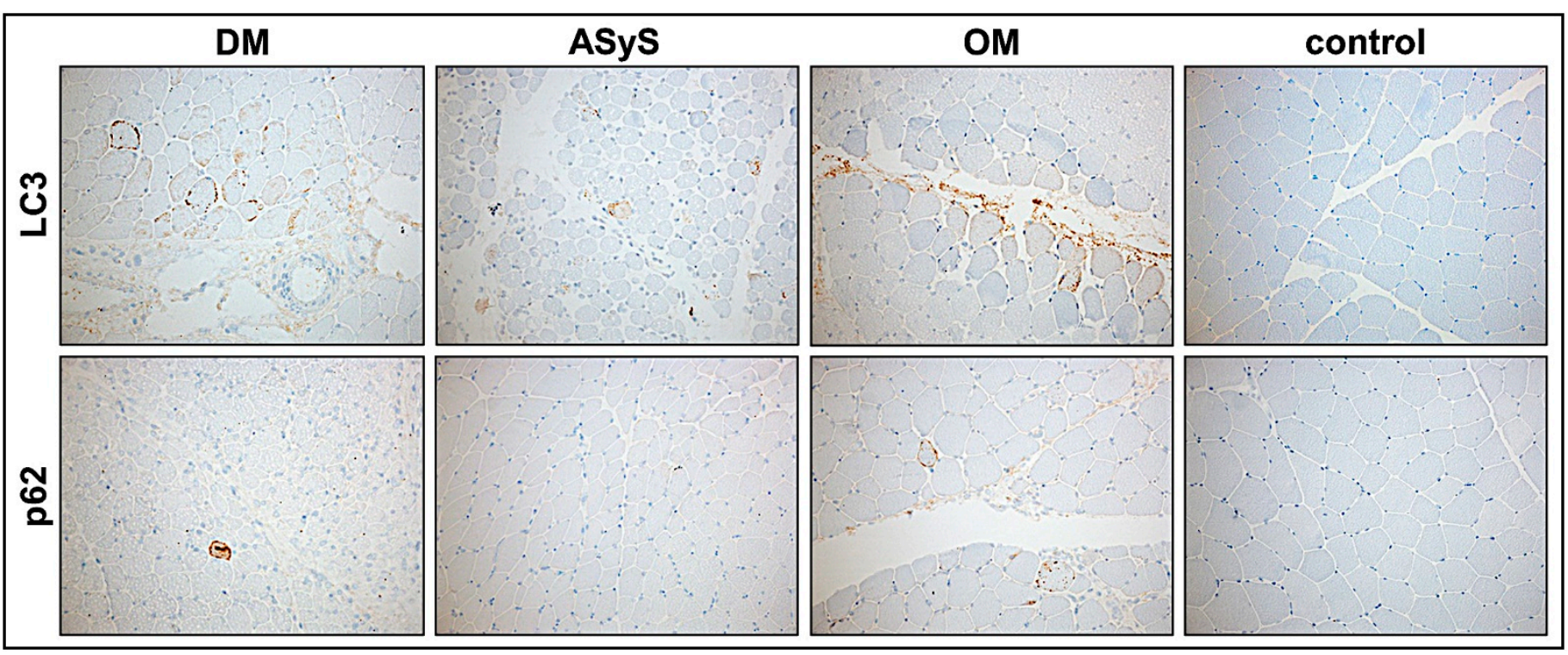

Figure 8. Expression of autopathic markers LC3 and p62 in jIIM muscle biopsies of patients with DM (P4), ASyS (P14), OM (P3), and control. Few muscle fibers expressing LC3 and p62 are present in DM (P4), ASyS (P14), and OM (P3), indicating a moderate upregulation of autophagy (magnification $20 \times) .(\mathrm{DM}=$ dermatomyositis; $\mathrm{ASyS}=$ antisynthetase syndrome; $\mathrm{OM}=$ overlap myositis $)$.

\subsubsection{Common Staining Pattern and Discriminative Key Findings in jIIM Subtypes}

Comparing the detailed analysis of thirty-one parameters shows common and distinct features, which are helpful to discriminate the different jIIM subtypes from each other. Muscle biopsies from controls showed a normal staining serving as a baseline. Common features in all jIIM muscle biopsies included the upregulation of MHC class I, lympho-monocyte cell infiltrates, scattered muscle fiber necrosis, and myofiber regeneration. Mitochondrial pathology with COX-negative fibers were exclusively present in DM. Anti-Jo-1-AsyS, but not PL-7-ASyS, shared some common features with DM, including perifascicular MxA upregulation, rarefication of CD31 ${ }^{+}$vessels and capillary C5b-9 deposits with endothelial inclusions (TIR), and punched-out vacuoles. Staining of the fragmented perimysium by ALP was present only in Jo-1-ASyS cases. Additional sarcolemmal C5b-9 deposits were more striking in Jo-1-ASyS. OM biopsies showed common morphological features of myositis, including with more scattered distribution compared to DM and anti-Jo-1-AsyS. Perifascicular atrophy can occur in $\mathrm{OM}$, but without perifascicular upregulation of MHC class 1 or MxA (Figure 9A,B). 


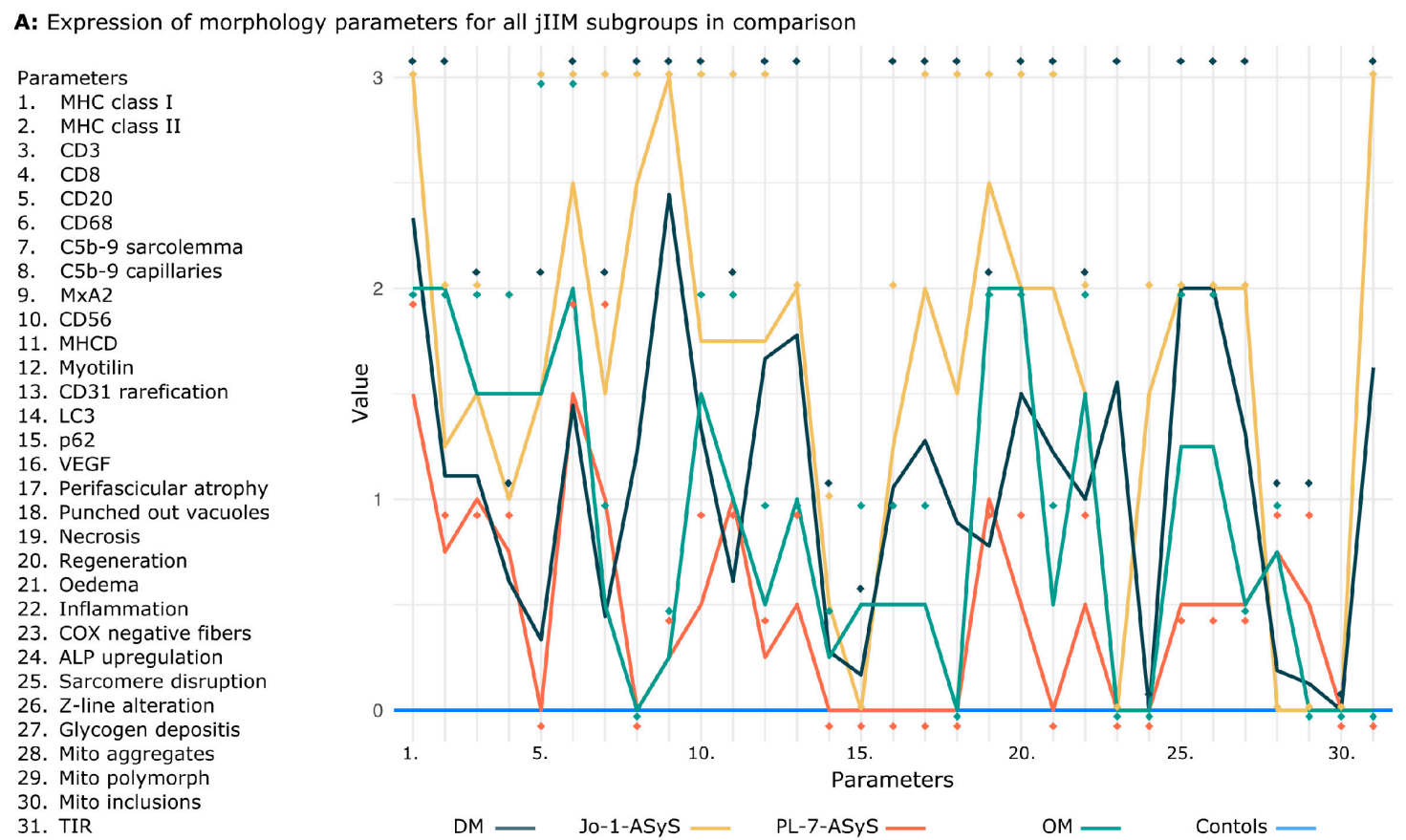

B: Expression of morphology parameters for each jIIM subgroup
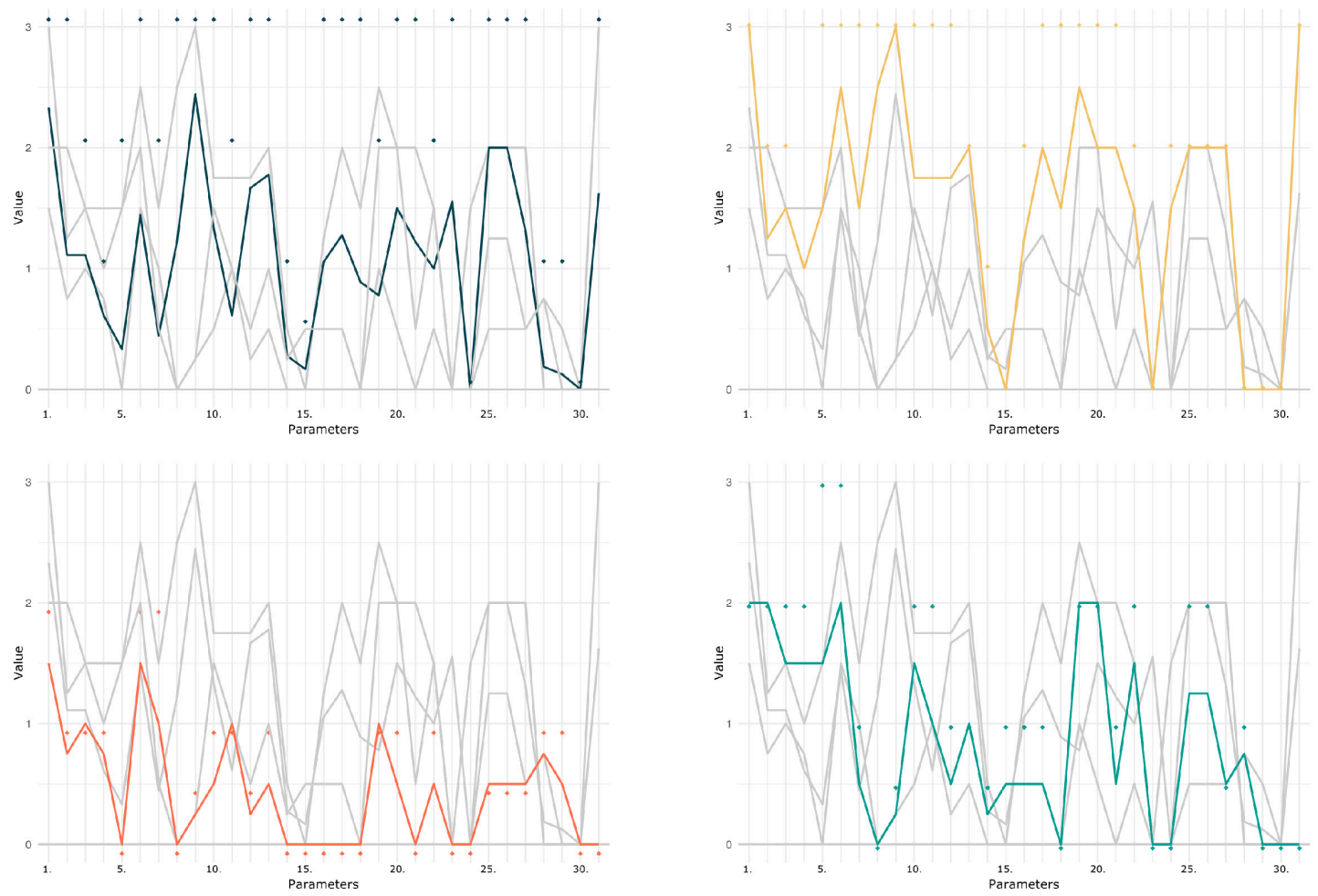

Figure 9. Visual exploration of the values. Common and discriminative morphological patterns in jIIM subgroups DM, ASyS, OM, and controls for 31 parameters, shown as mean (coloured lines) and maximum values (rhombus) per subgroup as an overview (A) and separated by subgroups (B). The minimum values per subgroup representing weak morphological characteristics are not shown. Healthy control (blue line) shows the normal pattern. Note the visible similar trend of the pattern of DM and Jo-1- ASyS compared to that in PL-7- ASyS and OM. For all parameters, the $p$-value did not reach statistical significance. (DM = dermatomyositis; ASyS = antisynthetase syndrome; $\mathrm{OM}=$ overlap myositis, $\mathrm{TIR}=$ tubuloreticular inclusions $)$. 


\section{Discussion}

The aim of this study was to analyze childhood myositis subtypes by using a comprehensive panel of stains that are widely available and recommended for diagnostic purposes in inflammatory myopathies [55]. This was carried out explicitly in a single tertiary center to avoid any confounders (including technical ones), and to gain full access to all additional data of the patients. Here, we describe that there are obvious and well-discernible morphological differences between DM and ASyS-associated myositis and overlap myositis in children. Those differences are well in line with current hypotheses about the different pathogeneses and the immune mechanisms involved in these entities. As we are approaching the era of targeted and individualized therapies, tailored according to the specific etiopathogenesis of diseases, it is of outmost importance to have a fine-tuned diagnostic repertoire that reflects immune pathogenesis in jIIM.

The juvenile patients enclosed in our study showed clinical signs of an IIM with the leading symptoms of subacute manifestation of proximal weakness and skin symptoms. The weakness was prevailing in the proximal lower legs with difficulties in climbing stairs. The diagnosis of jIIM was verified by MRI, EMG, and/or muscle ultrasound and laboratory data with an increased CK in the majority of patients. The distribution of age and gender with a higher proportion of affected females was in line with that of other studies [10,22]. Interestingly, in $33 \%$ of our young patients, sadness and mood swing were overt, which juxtaposes clinically reported signs of adult patients with IIM. Calcinosis, a skin symptom in jDM and uncommon in adult DM, was present in one third of our patients [24].

Compared to adults, the sub-classification of IIM in children is less well defined [3,4,6,54,56]. In our cohort, the majority of cases were diagnosed as DM, followed by ASyS and OM. MSA were analyzed in the majority of the patients, and in $50 \%$ of the patients, antibodies were detected, showing anti-NXP-2, anti-Jo1, and anti-PL-7 antibodies.

For morphological classification of juvenile IIM, only few studies were performed so far but without including all IIM subtypes and without using a specific and comprehensive myopathological repertoire of stains [33]. A common pathology in all of our juvenile IIM cases was the upregulation of MHC class 1 and MHC class 2, inflammation, oedema, necrotic fibers, and regeneration. Lympho-monocyte cell infiltrates were present in all subtypes. Compared to other studies, B-lymphocytes were not the predominant cell type in our DM cohort, but also occurred in Jo-1-ASyS and OM [57]. These analyses are mandatory to delineate IIM from hereditary myopathies. However, the staining alone does not discriminate between the distinct subtypes. Using detailed morphological analysis of thirtyone parameters showed a number of differences in staining patterns among the IIM subtypes.

Chronic disturbance in endothelial cell homeostasis, leading to a vasculopathy, is considered a major contributor to jDM, and markers of endothelial injury are increased in active jDM [58-60]. Severe vascular pathology predicts a chronic disease course and suggests poor prognostic factor in the outcome of jDM [60]. In muscle biopsies, a vasculopathy characterized by loss of endomysial vessels and hypoxia with upregulation of VEGF predominantly in perifascicular regions has been described with a strong heterogeneity in $\mathrm{jDM}$ patients [51].

Our data confirm the involvement of vascular pathology in jDM. A strong vasculopathy in jDM is associated with a reduced capillary network, capillary complement deposits, tubuloreticular endothelial inclusions, and upregulation of hypoxic marker (such as VEGF and strong muscle fiber pathology with disruption of the myofibrillar structure and hypoxia-driven pathology).

Vascular pathology was also present in anti-Jo-1-ASyS, leading to the hypothesis of a common pathway in pathogenesis compared to DM [39]. Vascular rarefication and VEGF upregulation also occurred in OM cases. These findings lead to the hypothesis that vascular involvement might play a role in pathogenesis, in inflammatory myopathies other than DM.

Microvascular membrane attack complex deposits in dermatomyositis might result from activation of the classical complement pathway triggered by direct binding of $\mathrm{C} 1 \mathrm{q}$ to injured endothelial cells [61]. In our cohort, capillary complement deposits (C5b-9) 
were present in patients with jDM and anti-Jo-1-ASyS. Sarcolemmal complement deposits were present with strong perifascicular expression in anti-Jo1-ASyS. This underlines the hypothesis that, in ASyS, complement decoration of myofibers is involved in interferon (IFN) $\gamma$-mediated myofiber damage in that specific area.

IFN-induced reactive oxygen species and mitochondrial damage contribute to muscle impairment and inflammation maintenance in dermatomyositis [62]. COX-deficient fibers are present in adult DM muscle samples with a variability different in MSA subgroups [56]. In our jIIM patients, COX-deficient fibers were exclusively present in DM muscles; thus, we can attach more importance to this staining for differentiating between certain subgroups. However, manifest mitochondrial ultrastructural alterations with formation of paracrystalline inclusions (as in monogenic mitochondriopathies or in IBM) were not seen.

Type I interferon (IFN) upregulation plays a key role in jIIM and inhibitory regulators of IFN, such as ISG15, as well as discriminated patients with DM from those with OM and inversely correlated with the severity of muscle pathology and positively with the clinical outcome [30]. The IFN surrogate marker, MxA, has a high specificity and sensitivity in DM cases and is recommended for muscle biopsy diagnosis by international consensus [54,63]. A perifascicular upregulation of MxA was seen in all of our jDM biopsies, with the exception of one case with an overall low pathology and sparse clinical symptoms. Strong MxA expression on muscle fibers and vessels was also present in one anti-Jo-1-ASyS case, but only weak or absent in anti-PL-7-ASyS. These data are in line with other studies, showing that MxA is expressed in a subset of AsyS cases. This suggests the possibility of common or overlapping pathological (type I and/or type II) interferon-related pathways in DM and ASyS, especially associated with anti-Jo1 antibodies [64]. This issue clearly warrants further detailed studies in the future. Endothelial inclusions (TIR) were also detected in the anti-Jo-1 ASyS, which is mainly a diagnostic criterion for DM, confirming this hypothesis.

In antisynthetase syndrome, our study highlighted the specific morphological features with perifascicular atrophy. As expected, the ALP staining pattern was strong in antiJo-1-ASyS and was not altered in other subgroups; thus, high sensitivity and specificity helps to distinguish ASyS from other myopathies, showing that ALP is a helpful diagnostic marker [39,52]. However, normal ALP staining does not exclude ASyS, but it is unlikely that it renders anti-Jo1-ASyS. The presence of filamentous nuclear inclusions are hallmarks of adult ASyS [41]. Only the specimen of an anti-Jo1-ASyS patient showed characteristic nuclear inclusions, whereas no nuclear inclusions were found in anti-PL-7-ASyS. From the data of our study, anti-Jo-1 and anti-PL-7-ASyS might have different pathological characteristics and should be further analyzed in larger cohorts.

In juvenile OM, the muscle pathology is not analyzed in detail so far. In our cohort, muscle pathology showed common features of IIM with MHC class I upregulation, inflammation, and oedema with a moderate pathology score. Tubuloreticular inclusions can occur in OM, especially in lupus erythematosus, but were not present in our cases $[65,66]$. MxA upregulation was weak on scattered fibers, but was not in a perifascicular distribution in OM, consistent with the upregulation of type I IFN in childhood SLE [18,67].

Autophagy is important for regulating homeostasis in cells. In skeletal and cardiac muscles, due to tension-induced force, misfolded proteins and damaged organelles are recycled by autophagic processes [68]. $\mathrm{P} 62^{+}$inclusion in myofibers are a nonspecific process following muscle injury, including myopathy and neurogenic atrophy, and is more prevalent in biopsies with more severe muscle damage [69]. In IIM, dysregulated or enhanced autophagy is described in IMNM and sIBM with distinct patterns of p62 inclusions and may play a role in the disease pathology [70]. In our study, the autophagic molecules, LC3 and p62, were visible only in few biopsies of DM, and anti-Jo-1-ASyS cases all showed higher scores of autophagy-related pathomorphology. This finding is consistent with an increased autophagy in more strongly affected muscle biopsies and awaits further detailed analysis in comparison to the adult situation.

For adults, a muscle biopsy to confirm the clinical diagnosis of myositis and characterize the myositis subtype is recommended by the German Neurological society, since a precise 
diagnosis is crucial for the therapy regime. Our data suggest that certain subtypes of myositis also exist in juvenile patients. Because these subtypes are less well defined compared to adults, and since myositis specific antibodies are false positive or missing in about $20 \%$ of children, we recommend a muscle biopsy in all juvenile patients with clinical symptoms of myositis. A small biopsy ( $0.5 \mathrm{~cm}$ in diameter) is adequate to perform a large set of staining and electron microscopy. Additionally, studies of larger cohorts are necessary to improve understanding on the underlying aetiopathology of juvenile myositis and therapy options.

\section{Limitations}

This study has some limitations. First, the clinical data from the patients were collected retrospectively. Therefore, MSA were not available for every patient in our cohort. Second, this is a single-center study which has certain advantages; however, the cohort of patients with such rare diseases is small, especially since the group of OM was diagnosed only in two patients with SLE. Therefore, the small sample size reduces the statistical power and the ability to formally identify significant effects in the cohort. Nevertheless, the described and visualized morphological and morphometric patterns of the subgroups provided very useful trajectories to define diagnostic and classification criteria.

\section{Conclusions}

Our study demonstrates the need of detailed and distinct morphological characterization of muscle pathology in juvenile myositis, with respect to precise prognostic and therapeutic decisions in jIIM, especially in children with negative or absent serological findings.

Supplementary Materials: The following are available online at https: / www.mdpi.com/article/ 10.3390/cells11010109/s1. Supplemental data. Table S1: Antibodies for IHC stains, Table S2: Detailed clinical data of patients with jIIM. Table S3: Laboratory data of patients with jIIM, Table S4: Electromyography, skeletal MRI and muscle ultrasound of patients with jIIM, Table S5: Morphology analysis of muscle biopsies from controls.

Author Contributions: Conceptualization, A.S. and W.S.; methodology, A.S., W.S., C.D., I.D., C.P. validation, A.S., W.S., H.-H.G.; investigation, L.R., A.H., writing-original draft preparation, A.S., W.S., C.P.; writing-review and editing, A.S., W.S., I.D., C.D., C.P., A.H., H.-H.G., A.D.M.; visualization, L.R., I.D.; All authors have read and agreed to the published version of the manuscript.

Funding: This research received no external funding.

Institutional Review Board Statement: The study was conducted according to the guidelines of the Declaration of Helsinki, and approved by the Institutional Review Board (or Ethics Committee) of Justus Liebig University Giessen, Germany (protocol code AZ07/09, AZ 258/16).

Informed Consent Statement: Informed consent was obtained from all subjects involved in the study.

Data Availability Statement: Data are available at supplemental data.

Acknowledgments: We thank the patients contributing to this study and Hannah Schlierbach, Angela Roth, Cordula zum Bruch and Kerstin Leib for their excellent technical assistance.

Conflicts of Interest: The authors declare no conflict of interest.

\section{Abbreviations}

ANA = antinuclear antibodies; $\mathrm{AP}=$ alkaline phosphatase; ASyS = anti-synthetase syndrome; $\mathrm{CK}=$ creatine kinase; $\mathrm{COX}=$ cytochrome oxidase; $\mathrm{DM}=$ dermatomyositis; IIM = idiopathic inflammatory myopathy; MxA = myxovirus resistance protein A; MSA = myositis specific antibodies; MAA = myositis-associated antibodies; MHC class $1=$ major histocompatibility factor class 1 ; $\mathrm{OM}=$ overlap myositis; $\mathrm{SLE}$ = systemic lupus erythematosus; $\mathrm{SDH}=$ succinate dehydrogenase; TEM = Transmission electron microscopy; TRI = tubuloreticular inclusions; VEGF = vascular endothelial growth factor. 


\section{Appendix A}

Table A1. Scoring of muscle pathology at standard staining.

\begin{tabular}{|c|c|c|c|c|c|c|c|c|c|c|}
\hline & \multirow[b]{2}{*}{ Diagnosis } & \multirow[b]{2}{*}{ VAS-Score } & \multicolumn{6}{|c|}{ HE } & \multirow{2}{*}{$\begin{array}{c}\text { COX-SDH } \\
\text { COX-Negative } \\
\text { Fibers }\end{array}$} & \multirow{2}{*}{$\begin{array}{c}\text { ALP } \\
\text { Upregulation }\end{array}$} \\
\hline & & & $\begin{array}{l}\text { Perifascicular } \\
\text { Atrophy (PA) }\end{array}$ & $\begin{array}{l}\text { Punched-Out- } \\
\text { Vacuoles } \\
\text { (POV) }\end{array}$ & Necrosis & Regeneration & Oedema & Inflammation & & \\
\hline P1 & $\mathrm{DM}$ & 4 & 1 & 0 & 1 & 2 & 1 & 1 & 2 & 0 \\
\hline P2 & DM & 6 & 3 & 1 & 0 & 3 & 1 & 1 & 3 & 0 \\
\hline P5 & DM & 6 & 1 & 3 & 1 & 1 & 3 & 2 & 3 & 0 \\
\hline P10 & DM & 4 & 0.5 & 0 & 1 & 1 & 1 & 0.5 & 0 & 0 \\
\hline P11 & DM & 1 & 0 & 0 & 0 & 0 & 0 & 0.5 & 0 & 0 \\
\hline $\mathrm{P} 12$ & $\mathrm{DM}$ & 8 & 3 & 1 & 2 & 3 & 2 & 2 & 3 & 0 \\
\hline P15 & DM & 2 & 0 & 0 & 1 & 0.5 & 1 & 0.5 & 0 & 0 \\
\hline $\mathrm{P} 4$ & $\begin{array}{c}\mathrm{DM} \\
(\mathrm{NXP}-2)\end{array}$ & 9 & 3 & 3 & 1 & 3 & 2 & 1 & 3 & 0 \\
\hline P13 & $\begin{array}{c}\mathrm{DM} \\
(\mathrm{NXP}-2)\end{array}$ & 1 & 0 & 0 & 0 & 0 & 0 & 0.5 & 0 & 0 \\
\hline P8 & $\begin{array}{l}\text { ASyS } \\
\text { (Jo-1) }\end{array}$ & 4 & 1 & 0 & 2 & 1 & 1 & 1 & 0 & 1 \\
\hline P14 & $\begin{array}{l}\text { ASyS } \\
\text { (Jo-1) }\end{array}$ & 9 & 3 & 3 & 3 & 3 & 3 & 2 & 0 & 2 \\
\hline P6 & $\begin{array}{l}\text { ASyS } \\
\text { (PL-7) }\end{array}$ & 2 & 0 & 0 & 1 & 0 & 0 & 1 & 0 & 0 \\
\hline P9 & $\begin{array}{l}\text { ASyS } \\
\text { (PL-7) }\end{array}$ & 2 & 0 & 0 & 1 & 1 & 0 & 0 & 0 & 0 \\
\hline P3 & $\begin{array}{c}\mathrm{OM} \\
\text { (SLE) }\end{array}$ & 4 & 1 & 0 & 2 & 2 & 0 & 1 & 0 & 0 \\
\hline P7 & $\begin{array}{l}\mathrm{OM} \\
\text { (SLE) }\end{array}$ & 6 & 0 & 0 & 2 & 2 & 1 & 2 & 0 & 0 \\
\hline
\end{tabular}

$\mathrm{DM}=$ dermatomyositis; ASyS = antisynthetase syndrome; OM = overlap myositis; SLE = systemic lupus erythematosus. 
Table A2. Scoring of muscle pathology at IH staining

\begin{tabular}{|c|c|c|c|c|c|c|c|c|c|c|c|c|c|c|c|c|c|}
\hline & \multirow{2}{*}{ Diagnosis } & \multicolumn{6}{|c|}{ Inflammation and Immunoactivation } & \multicolumn{2}{|c|}{$\begin{array}{l}\text { Complement } \\
\text { Deposits } \\
\text { (c5b-9) }\end{array}$} & \multirow{2}{*}{ MxA } & \multicolumn{2}{|c|}{$\begin{array}{l}\text { Regen- } \\
\text { Eration }\end{array}$} & \multirow{2}{*}{$\begin{array}{c}\text { Sarcomere } \\
\text { Disruption }\end{array}$} & \multicolumn{2}{|c|}{ Angiogenesis } & \multicolumn{2}{|c|}{ Autophagy } \\
\hline & & MHC1 & MHC2 & CD3 & CD8 & CD20 & CD68 & $\begin{array}{l}\text { Sarco- } \\
\text { Lemma }\end{array}$ & $\begin{array}{l}\text { Capil- } \\
\text { laries }\end{array}$ & & CD56 & MHCd & & CD31 Loss & VEGF & $\begin{array}{c}\mathrm{LC} \\
3\end{array}$ & p62 \\
\hline P1 & $\mathrm{DM}$ & 2 & 1 & 1 & 1 & 0 & 1 & 2 & 2 & 3 & 2 & 0 & 1 & 1 & 1 & 0 & 0 \\
\hline $\mathrm{P} 2$ & $\mathrm{DM}$ & 2 & 1 & 1 & 1 & 0 & 1 & 1 & 0 & 3 & 1 & 1 & 2 & 2 & 2 & 0 & 0 \\
\hline P5 & $\mathrm{DM}$ & 3 & 0.5 & 2 & 1 & 2 & 3 & 0 & 1 & 3 & 3 & 2 & 3 & 2 & 3 & 0.5 & 0.5 \\
\hline P10 & $\mathrm{DM}$ & 3 & 3 & 2 & 1 & 0 & 1 & 0 & 3 & 2 & 0 & 0 & 2 & 2 & 1 & 0 & 0 \\
\hline $\mathrm{P} 12$ & $\mathrm{DM}$ & 3 & 2 & 1 & 0 & 0 & 2 & 0 & 0.5 & 3 & 2 & 2 & 3 & 3 & 2 & 1 & 0.5 \\
\hline P15 & DM & 3 & 0.5 & 0.5 & 0.5 & 0 & 1 & 0 & 2 & 3 & 1 & 0 & 2 & 1 & 0 & 0 & 0 \\
\hline P4 & $\begin{array}{c}\text { DM } \\
(\mathrm{NXP}-2)\end{array}$ & 3 & 2 & 2 & 1 & 1 & 2 & 1 & 2 & 3 & 3 & 0.5 & 2 & 3 & 0.5 & 1 & 0.5 \\
\hline P13 & $\begin{array}{c}\text { DM } \\
\text { (NXP-2) }\end{array}$ & 0 & 0 & 0 & 0 & 0 & 1 & 0 & 0 & 0 & 0 & 0 & 0 & 1 & 0 & 0 & 0 \\
\hline P8 & $\begin{array}{l}\text { ASyS } \\
\text { (Jo-1) }\end{array}$ & 3 & 0.5 & 1 & 1 & 0 & 2 & 0 & 3 & 3 & 0.5 & 0.5 & 0.5 & 2 & 0.5 & 0 & 0 \\
\hline P6 & $\begin{array}{l}\text { ASyS } \\
\text { (PL-7) }\end{array}$ & 2 & 0.5 & 1 & 1 & 0 & 2 & 2 & 0 & 0.5 & 0 & 1 & 0 & 1 & 0 & 0 & 0 \\
\hline P9 & $\begin{array}{l}\text { ASyS } \\
\text { (PL-7) }\end{array}$ & 1 & 1 & 1 & 0.5 & 0 & 1 & 0 & 0 & 0 & 1 & 1 & 0.5 & 0 & 0 & 0 & 0 \\
\hline P3 & $\begin{array}{c}\mathrm{OM} \\
\text { (SLE) }\end{array}$ & 2 & 2 & 2 & 2 & 3 & 3 & 1 & 0 & 0.5 & 2 & 2 & 1 & 1 & 1 & 0.5 & 1 \\
\hline P7 & $\begin{array}{c}\mathrm{OM} \\
\text { (SLE) }\end{array}$ & 2 & 2 & 1 & 1 & 0 & 1 & 0 & 0 & 0 & 1 & 0 & 0 & 1 & 0 & 0 & 0 \\
\hline
\end{tabular}


Table A3. Scoring of muscle pathology at ultrastructural level.

\begin{tabular}{|c|c|c|c|c|c|c|c|c|c|}
\hline & \multirow{2}{*}{ Diagnosis } & \multicolumn{3}{|c|}{ Myofibrillar Structure } & \multicolumn{3}{|c|}{ Mitochondria } & \multirow{2}{*}{$\begin{array}{c}\begin{array}{c}\text { Endomysial } \\
\text { Capillaries }\end{array} \\
\text { Tubuloreticular } \\
\text { Structures (TIR) }\end{array}$} & \multirow{2}{*}{$\begin{array}{l}\text { Nuclear } \\
\text { Inclusions }\end{array}$} \\
\hline & & $\begin{array}{l}\text { Sarcomere Structure } \\
\text { Disruption }\end{array}$ & $\begin{array}{l}\text { Z-Line- } \\
\text { Alteration }\end{array}$ & $\begin{array}{l}\text { Glycogen } \\
\text { Deposits }\end{array}$ & Aggregation & Polymorphism & Inclusions & & \\
\hline P1 & $\mathrm{DM}$ & 1 & 1 & 2 & 1 & 1 & 0 & $\begin{array}{c}2 \\
(3 / 10)\end{array}$ & n.a. \\
\hline P2 & $\mathrm{DM}$ & 3 & 3 & 0 & 0.5 & 0 & 0 & $\begin{array}{c}2 \\
(4 / 10)\end{array}$ & n.a. \\
\hline P5 & $\mathrm{DM}$ & & & & n.a. & & & & \\
\hline P10 & $\mathrm{DM}$ & 2 & 2 & 2 & 0 & 0 & 0 & $\begin{array}{c}1 \\
(2 / 10)\end{array}$ & n.a. \\
\hline P11 & $\mathrm{DM}$ & 1 & 1 & 3 & 0 & 0 & 0 & $\begin{array}{c}3 \\
(6 / 10)\end{array}$ & n.a. \\
\hline P12 & $\mathrm{DM}$ & 3 & 3 & 0.5 & 0 & 0 & 0 & $\begin{array}{c}1 \\
(2 / 10)\end{array}$ & n.a. \\
\hline P15 & DM & 3 & 3 & 2 & 0 & 0 & 0 & $\begin{array}{c}1 \\
(2 / 10)\end{array}$ & n.a. \\
\hline $\mathrm{P} 4$ & $\begin{array}{c}\mathrm{DM} \\
(\mathrm{NXP}-2)\end{array}$ & 3 & 3 & 1 & 0 & 0 & 0 & $\begin{array}{c}3 \\
(8 / 10)\end{array}$ & n.a. \\
\hline P8 & $\begin{array}{l}\text { ASyS } \\
\text { (Jo-1) }\end{array}$ & & & & n.a. & & & & \\
\hline P14 & $\begin{array}{l}\text { ASyS } \\
\text { (Jo-1) }\end{array}$ & 2 & 2 & 2 & 0 & 0 & 0 & $\begin{array}{c}3 \\
(3 / 5)\end{array}$ & $3 / 410$ \\
\hline P6 & $\begin{array}{l}\text { ASyS } \\
\text { (PL-7) }\end{array}$ & 0.5 & 0.5 & 0.5 & 0.5 & 0 & 0 & $\begin{array}{c}0 \\
(0 / 10)\end{array}$ & $0 / 205$ \\
\hline P9 & $\begin{array}{l}\text { ASyS } \\
\text { (PL-7) }\end{array}$ & 0.5 & 0.5 & 0.5 & 1 & 1 & 0 & $\begin{array}{c}0 \\
(0 / 10)\end{array}$ & $0 / 210$ \\
\hline P3 & $\begin{array}{c}\mathrm{OM} \\
(\mathrm{SLE})\end{array}$ & 0.5 & 0.5 & 0.5 & 0.5 & 0 & 0 & $\begin{array}{c}0 \\
(0 / 10)\end{array}$ & n.a. \\
\hline P7 & $\begin{array}{l}\mathrm{OM} \\
(\mathrm{SLE})\end{array}$ & 2 & 2 & 0.5 & 1 & 0 & 0 & $\begin{array}{c}0 \\
(0 / 10)\end{array}$ & n.a. \\
\hline
\end{tabular}

n.a = not analyzed; DM = dermatomyositis; ASyS = antisynthetase syndrome; OM = overlap myositis; SLE = systemic lupus erythematodes. 


\section{References}

1. Ladislau, L.; Suarez-Calvet, X.; Toquet, S.; Landon-Cardinal, O.; Amelin, D.; Depp, M.; Rodero, M.P.; Hathazi, D.; Duffy, D.; Bondet, V.; et al. JAK inhibitor improves type I interferon induced damage: Proof of concept in dermatomyositis. Brain 2018, 141, 1609-1621. [CrossRef]

2. Melki, I.; Devilliers, H.; Gitiaux, C.; Bondet, V.; Belot, A.; Bodemer, C.; Quartier, P.; Crow, Y.J.; Duffy, D.; Rodero, M.P.; et al. Circulating Interferon- $\alpha$ Measured With a Highly Sensitive Assay as a Biomarker for Juvenile Inflammatory Myositis Activity: Comment on the Article by Mathian et al. Arthritis Rheumatol. 2020, 72, 195-197. [CrossRef] [PubMed]

3. Allenbach, Y.; Benveniste, O.; Goebel, H.H.; Stenzel, W. Integrated classification of inflammatory myopathies. Neuropathol. Appl. Neurobiol. 2017, 43, 62-81. [CrossRef]

4. De Bleecker, J.L.; De Paepe, B.; Aronica, E.; de Visser, M.; Amato, A.; Benveniste, O.; De Bleecker, J.; de Boer, O.; Dimachkie, M.; Gherardi, R.; et al. 205th ENMC International Workshop: Pathology diagnosis of idiopathic inflammatory myopathies part II, 28-30 March 2014, Naarden, The Netherlands. Neuromuscul. Disord. 2015, 25, 268-272. [CrossRef] [PubMed]

5. Mariampillai, K.; Granger, B.; Amelin, D.; Guiguet, M.; Hachulla, E.; Maurier, F.; Meyer, A.; Tohme, A.; Charuel, J.L.; Musset, L.; et al. Development of a New Classification System for Idiopathic Inflammatory Myopathies Based on Clinical Manifestations and Myositis-Specific Autoantibodies. JAMA Neurol. 2018, 75, 1528-1537. [CrossRef]

6. Tanboon, J.; Uruha, A.; Stenzel, W.; Nishino, I. Where are we moving in the classification of idiopathic inflammatory myopathies? Curr. Opin. Neurol. 2020, 33, 590-603. [CrossRef] [PubMed]

7. Siegert, E.; Uruha, A.; Goebel, H.H.; Preuße, C.; Casteleyn, V.; Kleefeld, F.; Alten, R.; Burmester, G.R.; Schneider, U.; Höppner, J.; et al. Systemic sclerosis-associated myositis features minimal inflammation and characteristic capillary pathology. Acta Neuropathol. 2021, 141, 917-927. [CrossRef]

8. Fredi, M.; Cavazzana, I.; Franceschini, F. The clinico-serological spectrum of overlap myositis. Curr. Opin. Rheumatol. 2018, 30, 637-643. [CrossRef]

9. Patwardhan, A.; Spencer, C.H. Biologics in refractory idiopathic inflammatory myositis (IIM): What experience in juvenile vs adult myositis tells us about the use of biologics in pediatric IIM. Mod. Rheumatol. 2021, 31, 933-948. [CrossRef]

10. Rider, L.G.; Nistala, K. The juvenile idiopathic inflammatory myopathies: Pathogenesis, clinical and autoantibody phenotypes, and outcomes. J. Intern. Med. 2016, 280, 24-38. [CrossRef]

11. Shah, M.; Mamyrova, G.; Targoff, I.N.; Huber, A.M.; Malley, J.D.; Rice, M.M.; Miller, F.W.; Rider, L.G. The clinical phenotypes of the juvenile idiopathic inflammatory myopathies. Medicine 2013, 92, 25-41. [CrossRef]

12. Bottai, M.; Tjärnlund, A.; Santoni, G.; Werth, V.P.; Pilkington, C.; de Visser, M.; Alfredsson, L.; Amato, A.A.; Barohn, R.J.; Liang, M.H.; et al. EULAR/ACR classification criteria for adult and juvenile idiopathic inflammatory myopathies and their major subgroups: A methodology report. RMD Open 2017, 3, e000507. [CrossRef]

13. Rider, L.G.; Shah, M.; Mamyrova, G.; Huber, A.M.; Rice, M.M.; Targoff, I.N.; Miller, F.W. The myositis autoantibody phenotypes of the juvenile idiopathic inflammatory myopathies. Medicine 2013, 92, 223-243. [CrossRef] [PubMed]

14. Ueki, M.; Kobayashi, I.; Takezaki, S.; Tozawa, Y.; Okura, Y.; Yamada, M.; Kuwana, M.; Ariga, T. Myositis-specific autoantibodies in Japanese patients with juvenile idiopathic inflammatory myopathies. Mod. Rheumatol. 2019, 29, 351-356. [CrossRef] [PubMed]

15. Yamasaki, Y.; Kobayashi, N.; Akioka, S.; Yamazaki, K.; Takezaki, S.; Nakaseko, H.; Ohara, A.; Nishimura, K.; Nishida, Y.; Sato, S.; et al. Clinical impact of myositis-specific autoantibodies on long-term prognosis of juvenile idiopathic inflammatory myopathies: Multicenter study. Rheumatology 2021, 60, 4821-4831. [CrossRef]

16. Betteridge, Z.E.; McHugh, N.J. Myositis-specific autoantibodies: An important tool to support diagnosis of myositis. J. Intern. Med. 2016, 280, 8-23. [CrossRef]

17. Bitencourt, N.; Solow, E.B.; Wright, T.; Bermas, B.L. Inflammatory myositis in systemic lupus erythematosus. Lupus 2020, 29 , 776-781. [CrossRef]

18. Turnier, J.L.; Pachman, L.M.; Lowe, L.; Tsoi, L.C.; Elhaj, S.; Menon, R.; Amoruso, M.C.; Morgan, G.A.; Gudjonsson, J.E.; Berthier, C.C.; et al. Comparison of Lesional Juvenile Myositis and Lupus Skin Reveals Overlapping Yet Unique Disease Pathophysiology. Arthritis Rheumatol. 2020, 73, 1062-1072. [CrossRef] [PubMed]

19. Kim, H.; Gunter-Rahman, F.; McGrath, J.A.; Lee, E.; de Jesus, A.A.; Targoff, I.N.; Huang, Y.; O'Hanlon, T.P.; Tsay, W.L.; Gadina, M.; et al. Expression of interferon-regulated genes in juvenile dermatomyositis versus Mendelian autoinflammatory interferonopathies. Arthritis Res. Ther. 2020, 6, 69. [CrossRef]

20. Kim, H.; Sanchez, G.A.; Goldbach-Mansky, R. Insights from Mendelian Interferonopathies: Comparison of CANDLE, SAVI with AGS, Monogenic Lupus. J. Mol. Med. 2016, 94, 1111-1127. [CrossRef]

21. Meyer, A.; Meyer, N.; Schaeffer, M.; Gottenberg, J.E.; Geny, B.; Sibilia, J. Incidence and prevalence of inflammatory myopathies: A systematic review. Rheumatology 2015, 54, 50-63. [CrossRef]

22. Tansley, S.L.; McHugh, N.J.; Wedderburn, L.R. Adult and juvenile dermatomyositis: Are the distinct clinical features explained by our current understanding of serological subgroups and pathogenic mechanisms? Arthritis Res. Ther. 2013, 15, 211. [CrossRef] [PubMed]

23. Wienke, J.; Deakin, C.T.; Wedderburn, L.R.; van Wijk, F.; van Royen-Kerkhof, A. Systemic and Tissue Inflammation in Juvenile Dermatomyositis: From Pathogenesis to the Quest for Monitoring Tools. Front. Immunol. 2018, 9, 2951. [CrossRef] 
24. Marhaug, G.; Shah, V.; Shroff, R.; Varsani, H.; Wedderburn, L.R.; Pilkington, C.A.; Brogan, P.A. Age-dependent inhibition of ectopic calcification: A possible role for fetuin-A and osteopontin in patients with juvenile dermatomyositis with calcinosis. Rheumatology 2008, 47, 1031-1037. [CrossRef] [PubMed]

25. Sag, E.; Demir, S.; Bilginer, Y.; Talim, B.; Haliloglu, G.; Topaloglu, H.; Ozen, S. Clinical features, muscle biopsy scores, myositis specific antibody profiles and outcome in juvenile dermatomyositis. Semin. Arthritis Rheum. 2021, 51, 95-100. [CrossRef]

26. Nguyen, M.; Do, V.; Yell, P.C.; Jo, C.; Liu, J.; Burns, D.K.; Wright, T.; Cai, C. Distinct tissue injury patterns in juvenile dermatomyositis auto-antibody subgroups. Acta Neuropathol. Commun. 2020, 8, 125. [CrossRef]

27. Li, L.; Liu, C.; Cheng, L.; Yan, S.; Chen, H.; Li, Y. Assessment of diagnostic utility, clinical phenotypic associations, and prognostic significance of anti-NXP2 autoantibody in patients with idiopathic inflammatory myopathies: A systematic review and meta-analysis. Clin. Rheumatol. 2021, 40, 819-832. [CrossRef]

28. Sanner, H.; Sjaastad, I.; Flato, B. Disease activity and prognostic factors in juvenile dermatomyositis: A long-term follow-up study applying the Paediatric Rheumatology International Trials Organization criteria for inactive disease and the myositis disease activity assessment tool. Rheumatology 2014, 53, 1578-1585. [CrossRef] [PubMed]

29. Bolko, L.; Jiang, W.; Tawara, N.; Landon-Cardinal, O.; Anquetil, C.; Benveniste, O.; Allenbach, Y. The role of interferons type I, II and III in myositis: A review. Brain Pathol. 2021, 31, e12955. [CrossRef]

30. Hou, C.; Durrleman, C.; Periou, B.; Barnerias, C.; Bodemer, C.; Desguerre, I.; Quartier, P.; Melki, I.; Rice, G.I.; Rodero, M.P.; et al. From Diagnosis to Prognosis: Revisiting the Meaning of Muscle ISG15 Overexpression in Juvenile Inflammatory Myopathies. Arthritis Rheumatol. 2021, 73, 1044-1052. [CrossRef] [PubMed]

31. Allenbach, Y.; Leroux, G.; Suarez-Calvet, X.; Preusse, C.; Gallardo, E.; Hervier, B.; Rigolet, A.; Hie, M.; Pehl, D.; Limal, N.; et al. Dermatomyositis With or Without Anti-Melanoma Differentiation-Associated Gene 5 Antibodies: Common Interferon Signature but Distinct NOS2 Expression. Am. J. Pathol. 2016, 186, 691-700. [CrossRef]

32. Hida, A.; Yamashita, T.; Hosono, Y.; Inoue, M.; Kaida, K.; Kadoya, M.; Miwa, Y.; Yajima, N.; Maezawa, R.; Arai, S.; et al. Anti-TIF1gamma antibody and cancer-associated myositis: A clinicohistopathologic study. Neurology 2016, 87, 299-308. [CrossRef]

33. Yasin, S.A.; Schutz, P.W.; Deakin, C.T.; Sag, E.; Varsani, H.; Simou, S.; Marshall, L.R.; Tansley, S.L.; McHugh, N.J.; Holton, J.L.; et al. Histological heterogeneity in a large clinical cohort of juvenile idiopathic inflammatory myopathy: Analysis by myositis autoantibody and pathological features. Neuropathol. Applied. Neurobiol. 2019, 45, 495-512. [CrossRef]

34. Noguchi, E.; Uruha, A.; Suzuki, S.; Hamanaka, K.; Ohnuki, Y.; Tsugawa, J.; Watanabe, Y.; Nakahara, J.; Shiina, T.; Suzuki, N.; et al. Skeletal Muscle Involvement in Antisynthetase Syndrome. JAMA Neurol. 2017, 74, 992-999. [CrossRef] [PubMed]

35. Hervier, B.; Benveniste, O. Clinical heterogeneity and outcomes of antisynthetase syndrome. Curr. Rheumatol. Rep. 2013, 15, 349. [CrossRef]

36. Gallay, L.; Gayed, C.; Hervier, B. Antisynthetase syndrome pathogenesis: Knowledge and uncertainties. Curr. Opin. Rheumatol. 2018, 30, 664-673. [CrossRef]

37. Gunawardena, H.; Betteridge, Z.E.; McHugh, N.J. Myositis-specific autoantibodies: Their clinical and pathogenic significance in disease expression. Rheumatology 2009, 48, 607-612. [CrossRef] [PubMed]

38. Rider, L.G.; Miller, F.W.; Targoff, I.N.; Sherry, D.D.; Samayoa, E.; Lindahl, M.; Wener, M.H.; Pachman, L.M.; Plotz, P.H. A broadened spectrum of juvenile myositis. Myositis-specific autoantibodies in children. Arthritis Rheum. 1994, 37, 1534-1538. [CrossRef]

39. Mescam-Mancini, L.; Allenbach, Y.; Hervier, B.; Devilliers, H.; Mariampillay, K.; Dubourg, O.; Maisonobe, T.; Gherardi, R.; Mezin, P.; Preusse, C.; et al. Anti-Jo-1 antibody-positive patients show a characteristic necrotizing perifascicular myositis. Brain 2015, 138, 2485-2492. [CrossRef]

40. Uruha, A.; Suzuki, S.; Suzuki, N.; Nishino, I. Perifascicular necrosis in anti-synthetase syndrome beyond anti-Jo-1. Brain 2016, 139, e50. [CrossRef]

41. Stenzel, W.; Preuße, C.; Allenbach, Y.; Pehl, D.; Junckerstorff, R.; Heppner, F.L.; Nolte, K.; Aronica, E.; Kana, V.; Rushing, E.; et al. Nuclear actin aggregation is a hallmark of anti-synthetase syndrome-induced dysimmune myopathy. Neurology 2015, 84 , 1346-1354. [CrossRef]

42. Allenbach, Y.; Benveniste, O.; Stenzel, W.; Boyer, O. Immune-mediated necrotizing myopathy: Clinical features and pathogenesis. Nat. Rev. Rheumatol. 2020, 16, 689-701. [CrossRef]

43. Mohassel, P.; Foley, A.R.; Donkervoort, S.; Fequiere, P.R.; Pak, K.; Bönnemann, C.G.; Mammen, A.L. Anti-3-hydroxy-3methylglutaryl-coenzyme a reductase necrotizing myopathy masquerading as a muscular dystrophy in a child. Muscle Nerve 2017, 56, 1177-1181. [CrossRef]

44. Tansley, S.L.; Betteridge, Z.E.; Simou, S.; Jacques, T.S.; Pilkington, C.; Wood, M.; Warrier, K.; Wedderburn, L.R.; McHugh, N.J. Anti-HMGCR Autoantibodies in Juvenile Idiopathic Inflammatory Myopathies Identify a Rare but Clinically Important Subset of Patients. J. Rheumatol. 2017, 44, 488-492. [CrossRef]

45. Kishi, T.; Rider, L.G.; Pak, K.; Barillas-Arias, L.; Henrickson, M.; McCarthy, P.L.; Shaham, B.; Weiss, P.F.; Horkayne-Szakaly, I.; Targoff, I.N.; et al. Association of Anti-3-Hydroxy-3-Methylglutaryl-Coenzyme A Reductase Autoantibodies With DRB1*07:01 and Severe Myositis in Juvenile Myositis Patients. Arthritis Care Res. 2017, 69, 1088-1094. [CrossRef]

46. Dubowitz, V.; Sewry, C.A.; Oldfords, A. Muscle Biopsy: A Practical Approach, 5th ed.; Elsevier Ltd.: Amsterdam, The Netherlands, 2021. 
47. Wedderburn, L.R.; Varsani, H.; Li, C.K.; Newton, K.R.; Amato, A.A.; Banwell, B.; Bove, K.E.; Corse, A.M.; Emslie-Smith, A.; Harding, B.; et al. International consensus on a proposed score system for muscle biopsy evaluation in patients with juvenile dermatomyositis: A tool for potential use in clinical trials. Arthritis Rheum. 2007, 57, 1192-1201. [CrossRef]

48. Varsani, H.; Charman, S.C.; Li, C.K.; Marie, S.K.; Amato, A.A.; Banwell, B.; Bove, K.E.; Corse, A.M.; Emslie-Smith, A.M.; Jacques, T.S.; et al. Validation of a score tool for measurement of histological severity in juvenile dermatomyositis and association with clinical severity of disease. Ann. Rheum. Dis. 2015, 74, 204-210. [CrossRef]

49. Kölbel, H.; Preuße, C.; Brand, L.; von Moers, A.; Della Marina, A.; Schuelke, M.; Roos, A.; Goebel, H.H.; Schara-Schmidt, U.; Stenzel, W. Inflammation, fibrosis and skeletal muscle regeneration in LGMDR9 are orchestrated by macrophages. Neuropathol. Appl. Neurobiol. 2021, 47, 856-866. [CrossRef]

50. Aschman, T.; Schneider, J.; Greuel, S.; Meinhardt, J.; Streit, S.; Goebel, H.H.; Büttnerova, I.; Elezkurtaj, S.; Scheibe, F.; Radke, J.; et al. Association Between SARS-CoV-2 Infection and Immune-Mediated Myopathy in Patients Who Have Died. JAMA Neurol. 2021, 78, 948-960. [CrossRef]

51. Preusse, C.; Allenbach, Y.; Hoffmann, O.; Goebel, H.H.; Pehl, D.; Radke, J.; Doeser, A.; Schneider, U.; Alten, R.H.; Kallinich, T.; et al. Differential roles of hypoxia and innate immunity in juvenile and adult dermatomyositis. Acta Neuropathol. Commun. 2016, 4, 45. [CrossRef]

52. Mozaffar, T.; Pestronk, A. Myopathy with anti-Jo-1 antibodies: Pathology in perimysium and neighbouring muscle fibres. J. Neurol. Neurosurg. Psychiatry 2000, 68, 472-478. [CrossRef] [PubMed]

53. Sallum, A.M.; Kiss, M.H.; Silva, C.A.; Wakamatsu, A.; Sachetti, S.; Lotufo, S.; Matsumura, N.; Marie, S.K. MHC class I and II expression in juvenile dermatomyositis skeletal muscle. Clin. Exp. Rheumatol. 2009, 27, 519-526.

54. Soponkanaporn, S.; Deakin, C.T.; Schutz, P.W.; Marshall, L.R.; Yasin, S.A.; Johnson, C.M.; Sag, E.; Tansley, S.L.; McHugh, N.J.; Wedderburn, L.R.; et al. Expression of myxovirus-resistance protein A: A possible marker of muscle disease activity and autoantibody specificities in juvenile dermatomyositis. Neuropathol. Appl. Neurobiol. 2019, 45, 410-420. [CrossRef] [PubMed]

55. Udd, B.; Stenzel, W.; Oldfors, A.; Olivé, M.; Romero, N.; Lammens, M.; Kusters, B.; Sewry, C.; Goebel, H.H.; Evangelista, T. 1st ENMC European meeting: The EURO-NMD pathology working group Recommended Standards for Muscle Pathology Amsterdam, The Netherlands, 7 December 2018. Neuromuscul. Disord. 2019, 29, 483-485. [CrossRef]

56. Benveniste, O.; Goebel, H.-H.; Stenzel, W. Biomarkers in Inflammatory Myopathies-An Expanded Definition. Front. Neurol. 2019, 10, 554. [CrossRef]

57. Sag, E.; Kale, G.; Haliloglu, G.; Bilginer, Y.; Akcoren, Z.; Orhan, D.; Gucer, S.; Topaloglu, H.; Ozen, S.; Talim, B. Inflammatory milieu of muscle biopsies in juvenile dermatomyositis. Rheumatol. Int. 2021, 41, 77-85. [CrossRef]

58. Baumann, M.; Gumpold, C.; Mueller-Felber, W.; Schoser, B.; Haberler, C.; Loescher, W.N.; Rostasy, K.; Fischer, M.B.; Wanschitz, J.V. Pattern of myogenesis and vascular repair in early and advanced lesions of juvenile dermatomyositis. Neuromuscul. Disord. 2018, 28, 973-985. [CrossRef]

59. Gitiaux, C.; De Antonio, M.; Aouizerate, J.; Gherardi, R.K.; Guilbert, T.; Barnerias, C.; Bodemer, C.; Brochard-Payet, K.; Quartier, P.; Musset, L.; et al. Vasculopathy-related clinical and pathological features are associated with severe juvenile dermatomyositis. Rheumatology 2016, 55, 470-479. [CrossRef]

60. Papadopoulou, C.; Hong, Y.; Krol, P.; Al Obaidi, M.; Pilkington, C.; Wedderburn, L.R.; Brogan, P.A.; Eleftheriou, D. The Vasculopathy of Juvenile Dermatomyositis: Endothelial injury, hypercoagulability, and increased arterial stiffness. Arthritis Rheumatol. 2021, 73, 1253-1266. [CrossRef]

61. Lahoria, R.; Selcen, D.; Engel, A.G. Microvascular alterations and the role of complement in dermatomyositis. Brain 2016, 139, 1891-1903. [CrossRef]

62. Meyer, A.; Laverny, G.; Allenbach, Y.; Grelet, E.; Ueberschlag, V.; Echaniz-Laguna, A.; Lannes, B.; Alsaleh, G.; Charles, A.L.; Singh F.; et al. IFN-beta-induced reactive oxygen species and mitochondrial damage contribute to muscle impairment and inflammation maintenance in dermatomyositis. Acta Neuropathol. 2017, 134, 655-666. [CrossRef]

63. Uruha, A.; Allenbach, Y.; Charuel, J.L.; Musset, L.; Aussy, A.; Boyer, O.; Mariampillai, K.; Landon-Cardinal, O.; Rasmussen, C.; Bolko, L.; et al. Diagnostic potential of sarcoplasmic MxA expression in subsets of dermatomyositis. Neuropathol. Appl. Neurobiol. 2019, 45, 513-522. [CrossRef]

64. Inoue, M.; Tanboon, J.; Okubo, M.; Theerawat, K.; Saito, Y.; Ogasawara, M.; Indrawati, L.A.; Uruha, A.; Okiyama, N.; Fujimoto, M.; et al. Absence of sarcoplasmic myxovirus resistance protein A (MxA) expression in antisynthetase syndrome in a cohort of 194 cases. Neuropathol. Appl. Neurobiol. 2019, 45, 523-524. [CrossRef]

65. Bronner, I.M.; Hoogendijk, J.E.; Veldman, H.; Ramkema, M.; van den Bergh Weerman, M.A.; Rozemuller, A.J.; de Visser, M. Tubuloreticular structures in different types of myositis: Implications for pathogenesis. Ultrastruct. Pathol. 2008, 32, 123-126. [CrossRef]

66. Carpenter, S.; Karpati, G.; Rothman, S.; Watters, G. The childhood type of dermatomyositis. Neurology 1976, $26,952-962$. [CrossRef]

67. Wong, D.; Kea, B.; Pesich, R.; Higgs, B.W.; Zhu, W.; Brown, P.; Yao, Y.; Fiorentino, D. Interferon and biologic signatures in dermatomyositis skin: Specificity and heterogeneity across diseases. PLoS ONE 2012, 7, e29161. [CrossRef]

68. Arndt, V.; Dick, N.; Tawo, R.; Dreiseidler, M.; Wenzel, D.; Hesse, M.; Fürst, D.O.; Saftig, P.; Saint, R.; Fleischmann, B.K.; et al. Chaperone-assisted selective autophagy is essential for muscle maintenance. Curr. Biol. 2010, 20, 143-148. [CrossRef] 
69. Milisenda, J.C.; Pinal-Fernandez, I.; Lloyd, T.E.; Grau, J.M.; Miller, F.W.; Selva-O'Callaghan, A.; Christopher-Stine, L.; Stenzel, W.; Mammen, A.L.; Corse, A.M. Accumulation of autophagosome cargo protein p62 is common in idiopathic inflammatory myopathies. Clin. Exp. Rheumatol. 2021, 39, 351-356.

70. Fischer, N.; Preusse, C.; Radke, J.; Pehl, D.; Allenbach, Y.; Schneider, U.; Feist, E.; von Casteleyn, V.; Hahn, K.; Ruck, T.; et al. Sequestosome-1 (p62) expression reveals chaperone-assisted selective autophagy in immune-mediated necrotizing myopathies. Brain Pathol. 2020, 30, 261-271. [CrossRef] 